medRxiv preprint doi: https://doi.org/10.1101/2020.04.16.20067975; this version posted April 22, 2020. The copyright holder for this preprint

(which was not certified by peer review) is the author/funder, who has granted medRxiv a license to display the preprint in perpetuity.

All rights reserved. No reuse allowed without permission.

\title{
LONG-TERM CLINICAL OUTCOMES IN SURVIVORS OF CORONAVIRUS OUTBREAKS AFTER HOSPITALISATION OR ICU ADMISSION: A SYSTEMATIC REVIEW AND META-ANALYSIS OF FOLLOW-UP STUDIES
}

Hassaan Ahmed ${ }^{1 *}$, Kajal Patel ${ }^{1 *}$, Darren Greenwood ${ }^{2,3}$, Stephen Halpin ${ }^{4,5,6}$, Penny Lewthwaite ${ }^{7}$, Abayomi Salawu $^{8}$, Lorna Eyre ${ }^{9}$, Andrew Breen ${ }^{9}$, Rory O'Connor ${ }^{4,5,6}$, Anthony Jones ${ }^{6}$, Manoj Sivan ${ }^{4,5,6}$

\section{Affiliated Institutions}

1 School of Medicine, University of Manchester, Manchester, UK.

2 School of Medicine, University of Leeds, Leeds, UK.

3 Leeds Institute for Data Analytics, University of Leeds, Leeds, UK.

4 Academic Department of Rehabilitation Medicine, University of Leeds

5 National Demonstration Centre of Rehabilitation Medicine, Leeds Teaching Hospitals NHS trust, Leeds, UK

6 Division of Neuroscience and Experimental Psychology, University of Manchester

7 Department of Infectious Diseases, Leeds Teaching Hospitals NHS Trust

8 Department of Rehabilitation Medicine, Hull University Teaching Hospitals NHS Trust

9 Intensive Care Unit, Leeds Teaching Hospitals NHS Trust

*Joint first authors

\section{Corresponding Author}

Name: Dr Manoj Sivan MD FRCP Ed

Address: Associate Professor and Honorary Consultant in Rehabilitation Medicine, Floor D, Martin Wing, Leeds General Infirmary, LS1 3EX.

E-mail address: m.sivan@leeds.ac.uk

Category: Systematic review and meta-analysis

Funding Sources: None

Conflict of Interest: None

Number of pages: 25

Number of references: 54

Number of tables: 6

Nymber of figures: 10 mew research that has not been certified by peer review and should not be used to guide clinical practice. 
medRxiv preprint doi: https://doi.org/10.1101/2020.04.16.20067975; this version posted April 22, 2020. The copyright holder for this preprint (which was not certified by peer review) is the author/funder, who has granted medRxiv a license to display the preprint in perpetuity. All rights reserved. No reuse allowed without permission.

\section{ABSTRACT}

Objective: To determine the long-term clinical problems in adult survivors of coronavirus (CoV) infection [Coronavirus disease 2019 (COVID-19), Severe Acute Respiratory Syndrome (SARS) and Middle East Respiratory Syndrome (MERS)] after hospitalisation or Intensive Care Unit (ICU) admission.

Design: Systematic review and meta-analysis of the literature.

Data sources: Ovid MEDLINE, EMBASE, CINAHL Plus and PsycINFO were searched using the strategy: (Coronavirus OR Coronavirus Infections OR COVID OR SARS virus OR Severe acute respiratory syndrome OR MERS OR Middle east respiratory syndrome) AND (Follow-up OR Follow-up studies OR Prevalence). Original studies reporting the clinical outcomes of adult survivors of coronavirus outbreaks two months after discharge or three months after admission were included. The quality of the studies was assessed using the Oxford Centre for Evidence-Based Medicine (OCEBM) 2009 Level of Evidence Tool. Meta-analysis was conducted to derive pooled estimates of prevalence and severity for different outcomes at time points up to 6 months follow-up and beyond 6 months follow-up.

Results: The search yielded 1169 studies of which 28 were included in this review. There were 15 Level 1b, 8 Level $2 b$, 2 Level $3 b$ and 3 Level 4 studies by OCEBM grading. Pooled analysis of studies revealed that complications commonly observed were impaired diffusing capacity for carbon monoxide (DLCO) [prevalence of $27.26 \%, 95 \% \mathrm{Cl} 14.87$ to 44.57 ] and reduced exercise capacity [(6-minute walking distance (6MWD) mean $461 \mathrm{~m}, 95 \% \mathrm{Cl} 449.66$ to 472.71 ] at 6 months with limited improvement beyond 6 months. Coronavirus survivors had considerable prevalence of psychological disorders such as post-traumatic stress disorder (PTSD) [38.80\%, Cl 30.93 to 47.31], depression [33.20\%, Cl 19.80 to 50.02] and anxiety [30.04\%, $\mathrm{Cl} 10.44$ to 61.26 ) beyond 6 months. These complications were accompanied by low Short Form 36 (SF-36) scores at 6 months and beyond indicating reduced quality of life which is present long-term.

Conclusions: The long term clinical problems in survivors of CoV infections (SARS and MERS) after hospitalisation or Intensive Care Unit (ICU) admission include respiratory dysfunction, reduced exercise capacity, psychological problems such as PTSD, depression and anxiety, and reduced quality of life. Critical care, rehabilitation and mental health services should anticipate a high prevalence of these problems following COVID-19 and ensure their adequate and timely management with the aim of restoring premorbid quality of life. 
medRxiv preprint doi: https://doi.org/10.1101/2020.04.16.20067975; this version posted April 22, 2020. The copyright holder for this preprint (which was not certified by peer review) is the author/funder, who has granted medRxiv a license to display the preprint in perpetuity. All rights reserved. No reuse allowed without permission.

\section{INTRODUCTION}

Coronavirus disease 2019 (COVID-19) is the third and largest outbreak of coronavirus (CoV) this century ${ }^{1}$. The disease is caused by severe acute respiratory syndrome coronavirus-2 (SARS-CoV$2)^{2}$ with the first cases reported in December $2019^{3}$. The World Health Organisation (WHO) declared the outbreak as a pandemic on 11th March $2020^{4}$, with currently more than 2 million infected cases reported worldwide ${ }^{5}$. Infection can lead to severe acute respiratory distress requiring critical care management, and case fatality is around $4 \%{ }^{6}$. As a result, much of the current effort is duly focused on improving mortality and ensuring intensive care units and hospital beds are not overwhelmed.

Coronavirus infection results in significant long-term morbidity not only through direct pathology, but also due to secondary disability and iatrogenic complications of treatments ${ }^{7}$. Even though these affect only some survivors ${ }^{7}$, the high prevalence of the disease means it will likely increase healthcare utilization significantly. Therefore, it is necessary to identify the prevalence of these long-term outcomes to facilitate timely preparations for the management of survivors. Whilst few studies are available yet on the long-term outcomes of COVID-19, the two previous CoV outbreaks $^{1}$ of Severe Acute Respiratory Syndrome (SARS) caused by SARS-CoV, originating in Guangdong, China in 2002, and Middle East Respiratory Syndrome (MERS) caused by MERS-CoV, originating in Saudi Arabia in 2012, could be used to model the longer-term impairments of the current pandemic.

This review aims to determine the long-term clinical complications in hospitalised survivors of SARS and MERS. The findings of this review will inform physicians about potential issues prevalent in survivors, help plan appropriate interventions and prepare health and social care services for subsequent increased healthcare utilization post-COVID-19.

\section{METHODS}

\section{Search Strategy}

A search of current literature was carried out in four databases - MEDLINE (1946 to March Week 3 2020), EMBASE (1974 to March 31 $\left.{ }^{\text {st }}, 2020\right)$, CINAHL Plus (1937 to March Week 32020 ) and PsycINFO (1806 to Match Week 3 2020). The search strategy used was: (Coronavirus OR Coronavirus Infections OR COVID OR SARS virus OR Severe acute respiratory syndrome OR MERS OR Middle east respiratory syndrome) AND (Follow-up OR Follow-up studies OR Prevalence). Terms were entered as MeSH terms where available for each database, otherwise these were searched as keywords in the title, abstract and subject headings. 
medRxiv preprint doi: https://doi.org/10.1101/2020.04.16.20067975; this version posted April 22, 2020. The copyright holder for this preprint (which was not certified by peer review) is the author/funder, who has granted medRxiv a license to display the preprint in perpetuity. All rights reserved. No reuse allowed without permission.

\section{Inclusion and Exclusion Criteria}

\section{Population}

Clinical studies involving adults with a confirmed diagnosis of coronavirus infection were included.

\section{Exposure}

Studies reporting patients with SARS, MERS or COVID-19 from current or previous outbreaks were included.

\section{Study Design}

Studies had to follow-up patients for a minimum period of 2 months post-discharge or 3 months postadmission to be included in this review. Only primary research studies were included. Reviews, casereports and editorial reports were excluded.

\section{Outcomes}

Studies were required to monitor changes in clinical symptoms at follow-up in order to be included. Studies which only monitored changes in serological or immunological results without any assessment of clinical status of the patient were excluded. Likewise, studies reporting only radiological appearance of lung disease or osteonecrosis without any mention of any clinical outcomes were also excluded

\section{Selection Process}

All studies were first screened using the title and abstract. At this stage abstracts with any mention of follow-up were included to avoid exclusion of abstracts which did not report the length of follow-up. Similarly, abstracts which reported follow-up for any outcome were accepted in order to allow inclusion of studies where clinical findings were not significant and therefore not reported in the abstract. Full texts of selected abstracts were then screened to ensure all the above selection criteria were met.

The finalised studies were then critically appraised and graded. Screening and grading were undertaken by four independent reviewers, KP, HA, MS and SH, and DG was involved in cases of disagreement.

\section{Data Extraction}

Data was extracted into standardised tables for each medical system. The following data was extracted: study, year, country, coronavirus outbreak, samples size, follow-up rate, age, sex, settings (hospital/ICU admission), follow-up period, prevalence of key outcomes, mean score for assessment 
medRxiv preprint doi: https://doi.org/10.1101/2020.04.16.20067975; this version posted April 22, 2020. The copyright holder for this preprint (which was not certified by peer review) is the author/funder, who has granted medRxiv a license to display the preprint in perpetuity. All rights reserved. No reuse allowed without permission.

of each outcome. Where estimates were only provided separately for 2 or more subgroups, we took the weighted average across those subgroups as the estimate for the overall population. Extraction was undertaken by at least two independent authors and further cross-checked by two more authors.

\section{Quality Assessment}

Studies were graded using the Oxford Centre for Evidence-Based Medicine (OCEBM) 2009 Level of Evidence Tool [Table 1$]^{8}$. The initial level of evidence was assigned depending on the type of study. Prospective cohort studies were then graded down if follow-up rate was $<80 \%$.

\section{Table 1. OCEBM Levels of Evidence}

\section{Level of Evidence Type of Study}

Level 1a

Systematic review of prospective cohort studies

Level $1 b$

Prospective cohort study with good follow-up ( $>80 \%)$

Level $1 \mathrm{c}$

All or none case-series

Level $2 a$

Systematic review of retrospective cohort study

Level $2 b$

Prospective cohort study with follow-up (<80\%) or Retrospective cohort study

Level $2 c$

Ecological studies

Level $3 a$

Systematic Review of Non-consecutive cohort study or very limited population

Level $3 b$

Non-consecutive cohort study

Level 4

Case-series

Level 5

Expert opinion without explicit critical appraisal, or based on physiology, bench research or "first principles"

\section{Data Analysis}

Binary data for prevalence of outcomes were pooled using meta-analysis by mixed-effects logistic regression. Mean scores for different outcomes were pooled in a meta-analysis using random effects models ${ }^{9}$. Forest plots were stratified by duration of follow-up (up to 6 months and over 6 months). Where a study presented more than one result within a subgroup, we selected the value closest to 6 months (for up to 6 months) or to 12 months (for over 6 months). Between-study heterogeneity was assessed as the range of study estimates, and the proportion of total variability attributable to between-study ${ }^{10}$. There were too few studies to formally explore the sources of heterogeneity through meta-regression (e.g. by mean age, disease, \% male, or level of evidence) or examine potential small-study effects such as publication bias through funnel plots. All statistical analyses were conducted using Stata version $15^{11}$. 
medRxiv preprint doi: https://doi.org/10.1101/2020.04.16.20067975; this version posted April 22, 2020. The copyright holder for this preprint (which was not certified by peer review) is the author/funder, who has granted medRxiv a license to display the preprint in perpetuity. All rights reserved. No reuse allowed without permission.

\section{RESULTS}

\section{Study Selection}

1169 studies were identified from the databases. Of these 104 abstracts were selected for full-text screening and finally 28 included in the review. The reasons for exclusion of the studies have been reported in Figure 1.

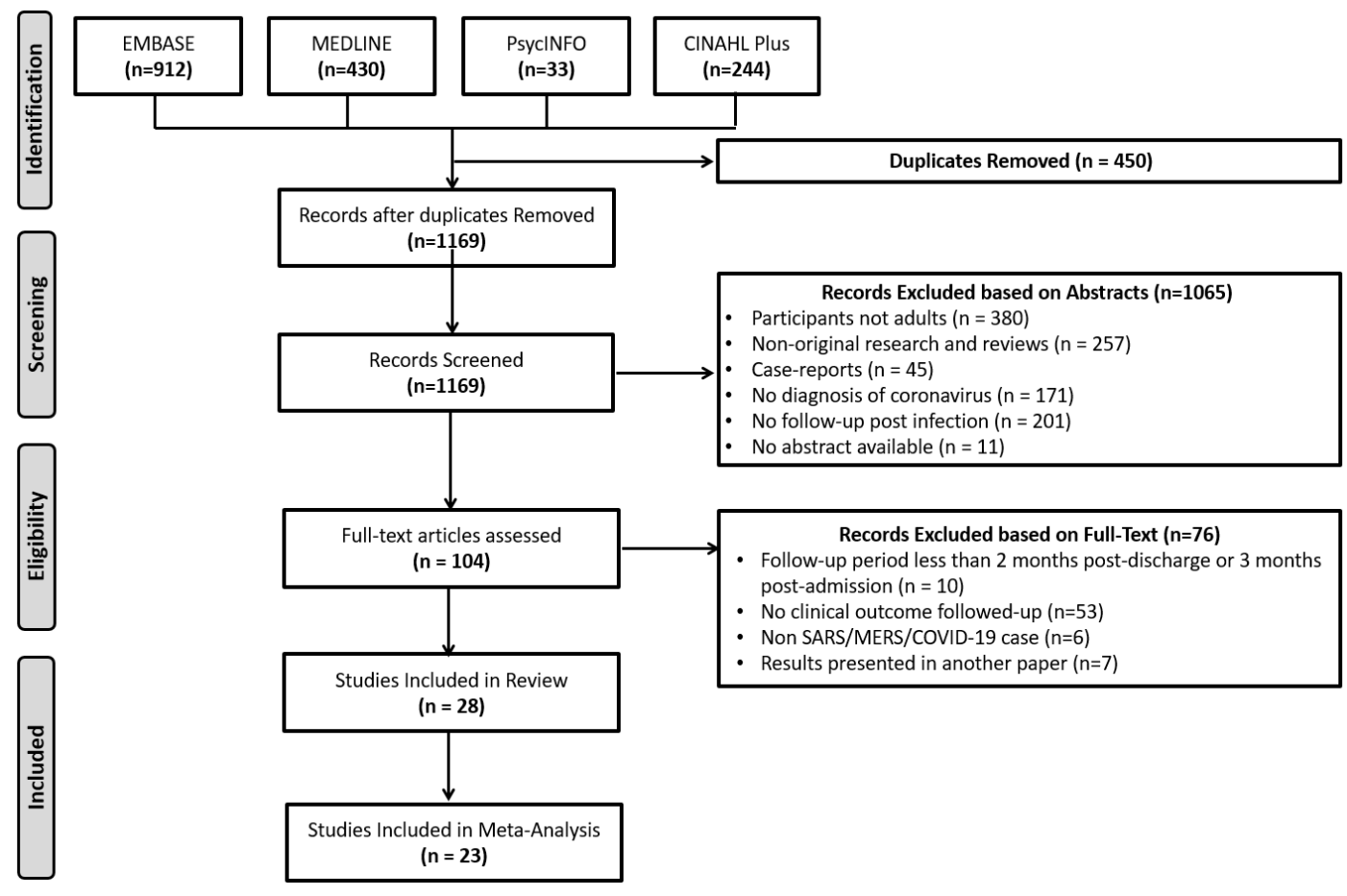

Figure 1. PRISMA Flowchart for the Literature Search

\section{Study Characteristics}

Out of the 28 studies included in this review, 26 studies reported findings from the SARS outbreak and 2 studies reported findings from the MERS outbreak. No studies have yet reported long-term outcomes of COVID-19 infection. The cohorts studied were from Beijing (11 studies), Hong Kong (9 studies), Guangzhou (1 study), Singapore (2 studies), Taiwan (2 studies), Korea (2 studies) and Canada (1 study) since these were the regions which have been severely affected by the previous outbreaks. The sample size ranged from a case series of 4 patients to a cohort study of 406 patients. There were 15 studies of Level 1b, 8 studies of Level 2b, 2 studies of Level 3b and 3 studies of Level 4 based on OCEBM grading. The 28 studies in the review reported outcomes involving multiple organ systems. The studies mainly addressed one or more of 5 key outcomes of interest - Lung function (18 studies), mental health ( 6 studies), exercise tolerance ( 5 studies), health-related quality of life (HRQoL) (5 studies), ocular (1 study) and neuromuscular outcomes (1 study). These are presented in Tables 2-6. 
TABLE 2. LUNG FUNCTION OUTCOMES

\begin{tabular}{|c|c|c|c|c|c|c|c|c|c|c|c|c|c|c|c|c|c|c|c|c|c|c|c|c|}
\hline $\begin{array}{l}\text { Stury } \\
\text { (vear) }\end{array}$ & $\begin{array}{c}\text { Coronanivs } \\
\text { ontutrecek }\end{array}$ & country & 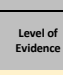 & 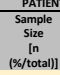 & 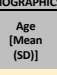 & 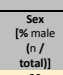 & Setting & $\begin{array}{c}\text { Follow } \\
\text { perod } \\
\text { Period }\end{array}$ & 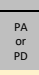 & 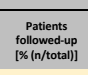 & $\begin{array}{l}\text { Prevalence of } \\
\text { wollocot } \\
{[\% \text { total]|] }}\end{array}$ & 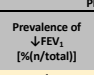 & 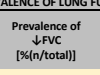 & 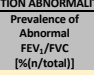 & 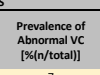 & 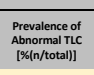 & 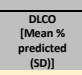 & 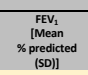 & 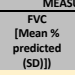 & 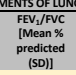 & 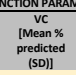 & 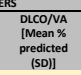 & 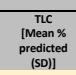 & 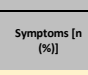 \\
\hline $\begin{array}{l}\text { Hee etal } \\
(2005)^{2}\end{array}$ & sars & Beijing & ${ }^{1 b}$ & 406 & 33(9) & $\begin{array}{l}201 \\
29056) \\
(92 / 45)\end{array}$ & Hosptal & 6 mon & PD & $994004 / 406$ & $\begin{array}{c}41 \\
(165 / 404)\end{array}$ & $\underset{(3 / 406)}{1}$ & & & {$[28 / 4006)$} & & & & & & & & & \\
\hline $\begin{array}{l}\text { chenent } \\
\text { (2006) } \\
(200)^{33}\end{array}$ & SARS & Bejing & 16 & 124 & ${ }_{40}$ & $\begin{array}{l}46 \\
(51 / 111)\end{array}$ & Hospital & $\begin{array}{l}3 \mathrm{mon} \\
18 \mathrm{mon}\end{array}$ & PD & $89(111 / 124)$ & & & & & & & & & & & & & & 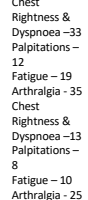 \\
\hline $\begin{array}{l}\text { Tanseyert } \\
(2007)^{4 *}\end{array}$ & SARS & Toronto & 1b & 117 & 42 & 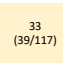 & Hospital & $\begin{array}{l}3 \text { mon } \\
6 \operatorname{mon} \\
12 \text { mon }\end{array}$ & PD & 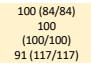 & & & & & & & $\begin{array}{l}87(1.1 .8) \\
86(.9) \\
85(8.9)\end{array}$ & $\begin{array}{l}107(19.3) \\
110(10.3) \\
109(192)\end{array}$ & $\begin{array}{l}98(1778) \\
103(177) \\
103(17.0)\end{array}$ & & & & 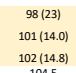 & \\
\hline 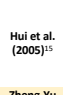 & SARS & Hong Kong & 1b & 110 & $37(10)$ & $\left(\begin{array}{l}400 \\
399 / 97)\end{array}\right.$ & $\begin{array}{c}\text { Hossitial } \\
\text { andicu }\end{array}$ & $\begin{array}{c}3 \text { mon } \\
6 \text { mon } \\
12 \text { mon }\end{array}$ & $\mathrm{PA}$ & $88(97 / 110)$ & $\begin{array}{r}13.5 \\
(13 / 97) \\
151 / 97) \\
(15 / 24) \\
(23 / 97)\end{array}$ & $\begin{array}{c}3 \\
(3 / 97) \\
44 / 97) \\
5 \\
5 / 97)\end{array}$ & $\begin{array}{c}6 \\
(6 / 97) \\
(4 / 97) \\
4 \\
(4 / 97)\end{array}$ & & $\begin{array}{c}6 \\
(6 / 97) \\
(5 / 997) \\
57 \\
1977\end{array}$ & $\begin{array}{l}7 \\
77 / 97) \\
(8 / 97) \\
(5 / 997)\end{array}$ & $\begin{array}{l}95.9(17.2) \\
95.5(19.4) \\
91.8(17.7)\end{array}$ & $\begin{array}{l}1075(14.6) \\
10068(14.9) \\
1065(14.7)\end{array}$ & 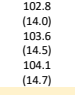 & & 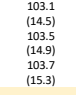 & & $\begin{array}{l}104.5 \\
118.0 \\
10.0 \\
106.7 \\
10.7) \\
105.8 \\
16.11\end{array}$ & \\
\hline 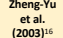 & sars & Beijing & $1 \mathrm{~b}$ & 100 & $37(11)$ & $\begin{array}{l}43 \\
(43 / 100)\end{array}$ & Hospital & 2 mon & PD & $91(191 / 100)$ & & & & & & & 80 & 93 & 96 & 83 & & & & \\
\hline 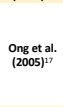 & SARS & Singapore & 1b & ${ }_{94}$ & $37(12)$ & $\begin{array}{l}26 \\
224 / 94)\end{array}$ & $\begin{array}{l}\text { HAspsital } \\
\text { and ICU }\end{array}$ & 12 mon & $\mathrm{PD}$ & ${ }_{10}^{10}(94 / 949)$ & $\begin{array}{c}213 \\
{[20 / 944)}\end{array}$ & $\begin{array}{l}221.34 \\
(201 / 94)\end{array}$ & $\begin{array}{l}12.8 \\
(12 / 994)\end{array}$ & ${ }_{(6,4 / 994)}^{64}$ & & $\begin{array}{c}8.54) \\
(8 / 94)\end{array}$ & $88.8(15.3)$ & $93.4(144)$ & $99.1(15.5)$ & $85.6(7.6)$ & $\begin{array}{l}103.0 \\
(15.0)\end{array}$ & $84.6(14.3)$ & $98.4(14.4)$ & 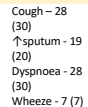 \\
\hline 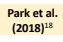 & MESS & Korea & ${ }^{1 b}$ & ${ }_{73}$ & $51(13)$ & $\underset{\substack{59 \\
437 / 73)}}{4}$ & Nation. & 12 mon & PD & $100(137 / 3)$ & $\begin{array}{r}34 \\
(25 / 73)\end{array}$ & ${ }_{(6, / 73)}^{8}$ & $\begin{array}{c}8 \\
(6 / 73)\end{array}$ & & & & $77(22)$ & $88(29)$ & $93(25)$ & $79(10)$ & & & $97(24)$ & \\
\hline$\underbrace{}_{\substack{\text { Lietetal } \\
(2006)^{9}}}$ & SAAS & Hong Kong & 1b & 45 & $42(12)$ & $\begin{array}{c}53 \\
(24 / 45)\end{array}$ & icu & $\begin{array}{l}3 \text { mon } \\
6 \operatorname{mon} \\
12 \text { mon }\end{array}$ & $\mathrm{PA}$ & $80(36 / 45)$ & & & & & & & $\begin{array}{l}78.5(14.5) \\
843.318 .3) \\
80.117 .5)\end{array}$ & $\begin{array}{l}989 .(11.4) \\
1002(14.8) \\
997(11.8)\end{array}$ & $\begin{array}{l}91.3(12.1) \\
95.8(14.0) \\
94.9(13.2)\end{array}$ & & & & $\begin{array}{l}92.6 .1(1.3) \\
108.17 \\
10.73 \\
87.8(13.9)\end{array}$ & \\
\hline 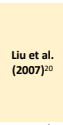 & SARS & Bejing & 1b & 37 & ${ }_{42(11)}$ & $\left\{\begin{array}{c}30 \\
(1 / 1 / 3)\end{array}\right.$ & Hospital & $\begin{array}{c}1 \mathrm{mon} \\
3 \mathrm{mon} \\
12 \mathrm{mon} \\
3 \mathrm{yss} .\end{array}$ & PD & $100(373737)$ & 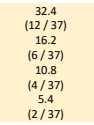 & 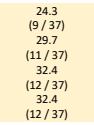 & 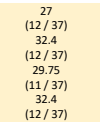 & 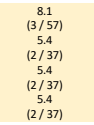 & & & $\begin{array}{l}80.9(37.5) \\
98.2(3.2) \\
107.3(33.3) \\
122.4(32.9)\end{array}$ & $\begin{array}{l}815(15.7) \\
845(14.4) \\
832(13.9) \\
85.1(15.8)\end{array}$ & $\begin{array}{l}78.2(13.7) \\
80.5(11.2) \\
81.2(16.3) \\
83.1(15.4)\end{array}$ & $\begin{array}{c}84.7(11.5) \\
84.99 .3) \\
84.1(10.4) \\
86.0(6.8)\end{array}$ & $\begin{array}{l}78.4(16.1) \\
79.8(13.5) \\
80.5(11.2) \\
81.1(15.6)\end{array}$ & & $\begin{array}{l}120.17 \\
151.1 \\
123.6 \\
143.6 \\
120.8 \\
140.83 \\
130.5 \\
(38.8)\end{array}$ & \\
\hline 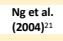 & SaAs & Hong Kong & $2 \mathrm{~b}$ & ${ }_{93}$ & $38(11)$ & ${ }_{(22 / 57)}^{39}$ & Hospital & 6 man & PA & $59(57 / 97)$ & $\begin{array}{r}35 \\
30157 \\
2015)\end{array}$ & $\begin{array}{l}16 \\
(9 / 57)\end{array}$ & $\begin{array}{l}127 / 7) \\
(4 / 57)\end{array}$ & & & $\begin{array}{l}30 \\
(117 / 57)\end{array}$ & & & & & & & & \\
\hline $\begin{array}{l}\text { 2hereget } \\
\text { (2005) } \\
\text { 2005) }\end{array}$ & SARS & $\begin{array}{l}\text { Suangzou } \\
\text { (China) }\end{array}$ & $2 b$ & 80 & $36(11)$ & $\begin{array}{c}38 \\
(10 / 26)\end{array}$ & Hosptatal & 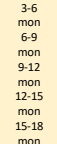 & PO & $33(26 / 800)$ & $(17 / 25)$ & & & & & & $\begin{array}{l}96(10) \\
96(16) \\
104(2) \\
93(21) \\
111(7)\end{array}$ & $\begin{array}{l}82(16) \\
91(12) \\
91(14) \\
94(18) \\
104(1)\end{array}$ & $\begin{array}{c}79(17) \\
92(13) \\
96(16) \\
93(17) \\
106(10)\end{array}$ & $\begin{array}{l}103(10) \\
105(6) \\
101(8) \\
106(4) \\
103(9)\end{array}$ & & $\begin{array}{c}69(15) \\
85(9) \\
102(18) \\
81(20) \\
118(21)\end{array}$ & $\begin{array}{c}67(21) \\
86(15) \\
96(20) \\
97(20) \\
114(33)\end{array}$ & \\
\hline 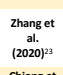 & SARS & Bejijng & 26 & 71 & Na & $\begin{array}{c}20 \\
(14 / 71)\end{array}$ & Hospital & $\begin{array}{l}\text { mon } \\
2 y s \\
15 y \mathrm{ys}\end{array}$ & PD & $\begin{array}{l}65(4 / 6 / 11) \\
73(52 / 71)\end{array}$ & 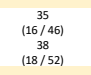 & & & ${ }_{11 / 52)}^{2}$ & & $\begin{array}{c}22 \\
(10 / 46) \\
0 / 52) \\
(0 / 52)\end{array}$ & $\begin{array}{l}83.9(10.2) \\
88.4(14.9)\end{array}$ & $\begin{array}{c}98.1(1.5) \\
1002(13.4)\end{array}$ & $\begin{array}{c}99.1116 .2) \\
10.33 \\
(14.8)\end{array}$ & $\begin{array}{l}84.9(6.1) \\
81.8(5.0)\end{array}$ & & $\begin{array}{l}94.8(11.6) \\
92.41(16.6)\end{array}$ & $\begin{array}{c}98.3(12.6) \\
102.6 \\
(12.6)\end{array}$ & \\
\hline $\begin{array}{l}\text { chinget } \\
\text { (2004) } \\
(2004)^{4}\end{array}$ & SAAS & Taivan & $2 b$ & 14 & $36(144)$ & ${ }^{21(3 / 1 / 4)}$ & Hospital & 6 mon & PA & ${ }^{649(9 / 14)}$ & $\begin{array}{l}50 \\
(5 / 9)\end{array}$ & & & & & & 69.420.3) & $802(15.6)$ & $76.9(15.2)$ & $103.5(7.4)$ & & & 92.19 .87 & \\
\hline 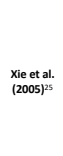 & SAAS & Beijing & 3b & 311 & $42(12)$ & $\begin{array}{l}34 \\
(29 / 55)\end{array}$ & Hospital & $\begin{array}{l}2 \mathrm{mon} \\
4 \mathrm{mon} \\
6 \mathrm{mon} \\
11 \mathrm{mon}\end{array}$ & PD & $13(40 / 311)$ & & & & & & & $\begin{array}{l}69(9) \\
76(11) \\
76(11) \\
79(12)\end{array}$ & $\begin{array}{l}83(13) \\
90(13) \\
93(12) \\
96(11)\end{array}$ & & & $\begin{array}{c}87(15) \\
94(14) \\
100(15) \\
103(15)\end{array}$ & $\begin{array}{l}95(14) \\
99(14) \\
97(14) \\
97(14)\end{array}$ & & \\
\hline 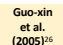 & SARS & Beijing & $3 b$ & 181 & 37(13) & $\begin{array}{c}41774 / \\
1811\end{array}$ & Hospital & 6 mon & PD & $\begin{array}{c}100 \\
(181 / 181)\end{array}$ & $\begin{array}{c}25 \\
46 / 1811\end{array}$ & & & & & & & & & & & & & \\
\hline 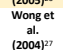 & SAAS & Hong Kong & зb & 99 & $39(13)$ & ${ }^{41}$ & Hospital & $\begin{array}{l}3 \text { mon } \\
6 \text { mon }\end{array}$ & ${ }^{\mathrm{PA}}$ & $\begin{array}{l}53(5487) \\
37(378)\end{array}$ & & & & & & & $\begin{array}{l}87.0(16.9) \\
88.020 .9)\end{array}$ & & & & & & & \\
\hline 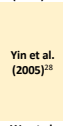 & SAAS & Beijng & ${ }^{4}$ & & ${ }_{42(15)}$ & ${ }^{20}$ & Hospital & $\begin{array}{l}1 \mathrm{mon} \\
3 \mathrm{mon} \\
6 \mathrm{mon} \\
12 \mathrm{mon}\end{array}$ & PD & 20 & 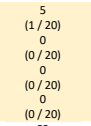 & $\begin{array}{c}15 \\
(3 / 20) \\
5 \\
5(20) \\
5 \\
(1 / 20) \\
001 \\
(0 / 20)\end{array}$ & & & & & $\begin{array}{l}83.9(0.0) \\
0.0(0.0) \\
0.0(0.0) \\
0.0(0.0)\end{array}$ & $\begin{array}{l}613(4.11) \\
67.0(0.0) \\
72(0.0) \\
0.0(0.0)\end{array}$ & & & & & & \\
\hline $\begin{array}{c}\text { Wuet al } \\
\text { (201615 } \\
\end{array}$ & SARS & Beijing & 4 & & $38(7)$ & 27 & Hospital & 7 years & $\mathrm{PD}$ & 11 & $\begin{array}{r}827 \\
(9 / 11) \\
\end{array}$ & & & & & & & & & & & & & \\
\hline
\end{tabular}


TABLE 3. EXERCISE TOLERANCE OUTCOMES

\begin{tabular}{|c|c|c|c|c|c|c|c|c|c|c|c|c|c|}
\hline \multicolumn{11}{|c|}{$\begin{array}{l}\text { PATIENT DEMOGRAPHICS } \\
\end{array}$} & \multicolumn{3}{|c|}{ EXERCISE OUTCOMES } \\
\hline Study (Year) & $\begin{array}{l}\text { Coronavirus } \\
\text { outbreak }\end{array}$ & Country & Level & $\begin{array}{l}\text { Sample } \\
\text { size } \\
{[\mathrm{n}]}\end{array}$ & $\begin{array}{c}\text { Age } \\
\text { [Mean } \\
\text { (SD)] }\end{array}$ & $\begin{array}{c}\text { Sex } \\
{[\% \text { male }} \\
(\mathrm{n} / \text { totall)] }\end{array}$ & Setting & $\begin{array}{l}\text { Follow-up } \\
\text { Period }\end{array}$ & $\begin{array}{l}\text { PA or } \\
\text { PD }\end{array}$ & $\begin{array}{l}\text { Patients followed- } \\
\text { up } \\
{[\% \text { (n/total) }]}\end{array}$ & $\begin{array}{l}\text { Exercise } \\
\text { test }\end{array}$ & $\begin{array}{l}\text { Prevalence }[\%(\mathrm{n} / \text { totall)] of } \\
\text { reduced V02max }\end{array}$ & $\begin{array}{l}\text { 6MWD (m) } \\
\text { [Mean (SD)] }\end{array}$ \\
\hline \multirow{3}{*}{ Hui et al. (2005) ${ }^{15}$} & \multirow{3}{*}{ SARS } & \multirow{3}{*}{ Hong Kong } & \multirow{3}{*}{$1 \mathrm{~b}$} & \multirow{3}{*}{110} & \multirow{3}{*}{$37(10)$} & \multirow{3}{*}{$40(39 / 97)$} & \multirow{3}{*}{$\begin{array}{l}\text { Hospital and } \\
\text { ICU }\end{array}$} & 3 mon & PA & $88(97 / 110)$ & 6MWD & N/A & $464(87)^{*}$ \\
\hline & & & & & & & & \multirow{2}{*}{$12 \mathrm{mon}$} & PA & $88(97 / 110)$ & 6MWD & $\mathrm{N} / \mathrm{A}$ & $502(97) * \#$ \\
\hline & & & & & & & & & PA & $88(97 / 110)$ & 6MWD & N/A & $511(90) * \#$ \\
\hline Park et al. (2018) ${ }^{18}$ & MERS & $\begin{array}{l}\text { Republic/ } \\
\text { Korea }\end{array}$ & $1 \mathrm{~b}$ & 73 & $51(13)$ & $60(43 / 73)$ & Hospital & $12 \mathrm{mon}$ & PD & $100(73 / 73)$ & 6MWD & $\mathrm{N} / \mathrm{A}$ & $540(172)$ \\
\hline Ong et al. (2004) & SARS & Singapore & $1 \mathrm{~b}$ & 46 & $37(11)$ & $26(12 / 44)$ & $\begin{array}{l}\text { Hospital and } \\
\text { ICU }\end{array}$ & $3 \mathrm{mon}$ & PD & $96(44 / 46)$ & CPET & $41(18 / 44)$ & $\mathrm{N} / \mathrm{A}$ \\
\hline \multirow{3}{*}{ Li et al. (2006) } & \multirow{3}{*}{ SARS } & \multirow{3}{*}{ Hong Kong } & \multirow{3}{*}{$1 \mathrm{~b}$} & \multirow{3}{*}{36} & \multirow{3}{*}{$42(12)$} & \multirow{3}{*}{$54(24 / 80)$} & \multirow{3}{*}{ ICU } & 3 mon & PA & $100(36 / 36)$ & 6MWD & N/A & $454(98)$ \\
\hline & & & & & & & & 6 mon & PA & $100(36 / 36)$ & 6MWD & $\mathrm{N} / \mathrm{A}$ & $504(107)$ \\
\hline & & & & & & & & $12 \mathrm{mon}$ & PA & $100(36 / 36)$ & 6MWD & $\mathrm{N} / \mathrm{A}$ & $506(111)$ \\
\hline \multirow[b]{2}{*}{ Lam et al. (2006) $)^{31}$} & \multirow[b]{2}{*}{ SARS } & \multirow[b]{2}{*}{ Hong Kong } & \multirow[b]{2}{*}{$2 \mathrm{~b}$} & \multirow[b]{2}{*}{116} & \multirow[b]{2}{*}{$46(15)$} & \multirow[b]{2}{*}{$44(51 / 81)$} & \multirow[b]{2}{*}{ Hospital } & 2 mon & PA & $70(81 / 116)$ & 6MWD & N/A & 468 (111) \\
\hline & & & & & & & & $8 \mathrm{mon}$ & PA & $70(81 / 116)$ & 6MWD & N/A & $577(96)$ \\
\hline
\end{tabular}

$>3$ months) 
TABLE 4. MENTAL HEALTH OUTCOMES

\begin{tabular}{|c|c|c|c|c|c|c|c|c|c|c|c|c|c|c|c|c|c|c|c|}
\hline \multicolumn{10}{|c|}{ PATIENT DEMOGRAPHICS } & \multicolumn{6}{|c|}{ PREVALENCE OF MENTAL HEALTH OUTCOMES } & \multicolumn{4}{|c|}{ MEAN SCORES FOR MENTAL HEALTH OUTCOMES } \\
\hline $\begin{array}{l}\text { Study } \\
\text { (Year) }\end{array}$ & $\begin{array}{l}\text { Coronavirus } \\
\text { outbreak }\end{array}$ & Country & $\begin{array}{l}\text { Level of } \\
\text { Evidence }\end{array}$ & $\begin{array}{c}\text { Sample } \\
\text { Size } \\
{[\mathrm{n}]}\end{array}$ & $\begin{array}{c}\begin{array}{c}\text { Age } \\
\text { [Mean } \\
\text { (SD)] }\end{array} \\
\end{array}$ & $\begin{array}{l}\text { Sex } \\
{[\% \text { male }} \\
[\mathrm{n} / \text { total })]\end{array}$ & Setting & $\begin{array}{l}\text { Follow- } \\
\text { up } \\
\text { Period }\end{array}$ & $\begin{array}{l}\text { PA } \\
\text { or } \\
\text { PD }\end{array}$ & $\begin{array}{c}\text { Patients } \\
\text { followed-up } \\
{[\%} \\
\text { [n/total)] }\end{array}$ & $\begin{array}{c}\text { Prevalence of } \\
\text { PPSD } \\
{[\%(n / \text { totall)] }}\end{array}$ & $\begin{array}{l}\text { Prevalence of } \\
\text { Depression } \\
{[\%(n / t \text { totall)] }}\end{array}$ & $\begin{array}{l}\begin{array}{l}\text { Prevalence of } \\
\text { Anxiett } \\
{[\%(h / \text { totall)] }}\end{array}\end{array}$ & $\begin{array}{l}\text { Prevalence of } \\
\text { Crronic Fatigue } \\
\text { [\%(n/totalal)] }\end{array}$ & $\begin{array}{l}\text { Prevalence of } \\
\text { Pain Disorder } \\
[\%(n)]]\end{array}$ & $\begin{array}{c}\text { Mean PTSD } \\
\text { score } \\
\text { [Mean (SD)] }\end{array}$ & $\begin{array}{c}\text { Mean } \\
\text { Depression } \\
\text { Score [mean } \\
\text { [SDD)] }\end{array}$ & $\begin{array}{c}\text { Mean } \\
\text { Anxiety } \\
\text { Score } \\
\text { [mean } \\
\text { (SD)] } \\
\end{array}$ & $\begin{array}{c}\text { Mean } \\
\text { Fatigue } \\
\text { Score } \\
\text { [mean } \\
(\mathrm{SD})] \\
\end{array}$ \\
\hline $\begin{array}{l}\text { Lee et al. } \\
(2007)^{2}\end{array}$ & SARS & $\begin{array}{l}\text { Hong } \\
\text { Kong }\end{array}$ & $1 \mathrm{~b}$ & 120 & NA & $36(35 / 96)$ & Hospital & 12 mon & PD & $\begin{array}{c}80 \% \\
(96 / 120)\end{array}$ & $\begin{array}{c}32.2(25 / 79) \\
\text { (IIS-R) }\end{array}$ & $\begin{array}{c}40.7(32 / 79) \\
\text { (DASS) }\end{array}$ & $\begin{array}{c}51.5(41 / 79) \\
\text { (DASS) }\end{array}$ & & & $\begin{array}{l}1.4(0.9) \\
(\text { (IES-R) }\end{array}$ & $\begin{array}{c}11.1(9.1) \\
\text { (DASS) }\end{array}$ & $\begin{array}{l}10.4(8.0 \\
\text { (DASS) }\end{array}$ & \\
\hline $\begin{array}{l}\text { Mak et al. } \\
(2009)^{33}\end{array}$ & SARS & $\begin{array}{l}\text { Hong } \\
\text { Kong }\end{array}$ & $1 \mathrm{~b}$ & 93 & $41(12)$ & $38(34 / 90)$ & Hospital & $2.5 \mathrm{yrs}$ & PA & $\begin{array}{l}96.8(90 / \\
93)\end{array}$ & $\begin{array}{c}25.6(23 / 90) \\
(\text { (IIS-R) }\end{array}$ & 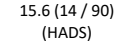 & 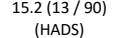 & & & & & & \\
\hline $\begin{array}{l}\text { Gao et al. } \\
(2006)^{34}\end{array}$ & SARS & Bejijng & $1 \mathrm{~b}$ & 76 & $25(9)$ & $31(21 / 67)$ & Hospital & $\begin{array}{c}3 \text { mon } \\
12 \text { mon }\end{array}$ & PD & $88(67 / 76)$ & $\begin{array}{l}46.2(31 / 67) \\
38.8(26 / 67) \\
\text { (PTSD-SS) }\end{array}$ & & & & & & & & \\
\hline \multirow{5}{*}{ 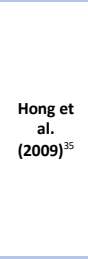 } & \multirow{5}{*}{ SARS } & \multirow{5}{*}{ Beijing } & \multirow{5}{*}{${ }^{1 b}$} & \multirow{5}{*}{70} & \multirow{5}{*}{$39(12)$} & \multirow{5}{*}{$33(23 / 70)$} & \multirow{5}{*}{ Hospital } & 2 mon & \multirow{5}{*}{ PD } & $\begin{array}{c}100(70 / \\
70)\end{array}$ & $40.0(28 / 70)$ & & & & & $32.0(18.3)$ & $39.5(12,5)$ & $\begin{array}{l}33.3 \\
(15.9)\end{array}$ & \\
\hline & & & & & & & & 7 mon & & $86(60 / 70)$ & $41.0(25 / 60)$ & & & & & $25.5(16.5)$ & $38.9(13.0)$ & $\begin{array}{l}30.5 \\
(12.9)\end{array}$ & \\
\hline & & & & & & & & 10 mon & & $81(57 / 70)$ & $38.6(22 / 57)$ & & & & & $27.9(19.9)$ & $37.5(13.1)$ & $\begin{array}{l}30.1 \\
(12.6)\end{array}$ & \\
\hline & & & & & & & & 20 mon & & $83(58 / 70)$ & $39.7(23 / 58)$ & & & & & $22.3(20.1)$ & $33.1(12.8)$ & $\begin{array}{l}29.0 \\
(13.4)\end{array}$ & \\
\hline & & & & & & & & 4 yrs & & $83(57 / 70)$ & $\begin{array}{l}42.1(24 / 58) \\
\text { (CCMD-III) }\end{array}$ & & & & & $\begin{array}{c}22.1(21.2) \\
(\text { (IES-R) }\end{array}$ & $\begin{array}{c}32.4(15.0) \\
\text { (SDS) }\end{array}$ & $\begin{array}{l}28.8 \\
(11.3) \\
(\mathrm{SAS})\end{array}$ & \\
\hline \multirow{2}{*}{$\begin{array}{l}\text { Lee et al. } \\
(2019)^{16}\end{array}$} & \multirow{2}{*}{ MERS } & \multirow{2}{*}{ Korea } & \multirow{2}{*}{$1 \mathrm{~b}$} & \multirow{2}{*}{72} & \multirow{2}{*}{$50(12)$} & \multirow{2}{*}{$62(32 / 52)$} & \multirow{2}{*}{ Hospital } & 12 mon & \multirow{2}{*}{ PD } & $88(63 / 72)$ & $42.3(27 / 63)$ & $26.9(17 / 63)$ & & $48.1(30 / 63)$ & & $\begin{array}{c}25.8(20.0) \\
\text { (IES-R) }\end{array}$ & $6.5(5.7)$ & & $3.5(1.9)$ \\
\hline & & & & & & & & 18 mon & & $75(54 / 72)$ & $\begin{array}{l}26.9(15 / 54) \\
(\text { (IES-R) }\end{array}$ & $\begin{array}{c}17.3(9 / 54) \\
\text { (PHQ-9) }\end{array}$ & & $\begin{array}{l}32.7(18 / 54) \\
\text { (FSS) }\end{array}$ & & $\begin{array}{c}19.3(21.0) \\
(1 E S-R)\end{array}$ & $\begin{array}{l}5.4(5.7) \\
(\mathrm{POO}-9)\end{array}$ & & $\begin{array}{c}2.8(1.9) \\
\text { (lfs5) }\end{array}$ \\
\hline $\begin{array}{l}\text { Lam et tal. } \\
(2009)^{37}\end{array}$ & SARS & $\begin{array}{l}\text { Hong } \\
\text { Kong }\end{array}$ & $2 \mathrm{~b}$ & 233 & $43(14)$ & $\begin{array}{c}30 \\
(69 / 233)\end{array}$ & Hospital & $3.5 \mathrm{yrs}$ & PD & $\begin{array}{c}78(181 / \\
233)\end{array}$ & $\begin{array}{c}54.5 \\
(127 / 233) \\
(I E S-R)\end{array}$ & $\begin{array}{c}39 \\
\left(\begin{array}{c}31 / 233) \\
\text { (HADS) }\end{array}\right.\end{array}$ & & $\begin{array}{l}40.3(94 / 233) \\
(\mathrm{COCO}) \\
27.1(63) / 233) \\
(\mathrm{CDCC})\end{array}$ & $\begin{array}{c}36.4 \\
(85 / 233)\end{array}$ & $\begin{array}{l}1.6(1.0) \\
(I E S-R)\end{array}$ & $\begin{array}{l}7.44 .5) \\
\text { (HADS) }\end{array}$ & $\begin{array}{l}7.6(4.4) \\
\text { (HADS) }\end{array}$ & \\
\hline
\end{tabular}

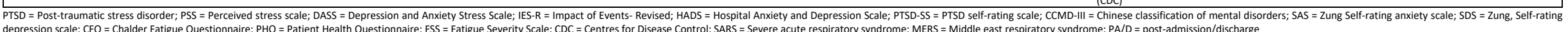
depression scale; CFQ = Chalder Fatigue Questionnaire; PHQ = Patient Health Questionnaire; FSS = Fatigue Severity Scale; CDC = Centres for Disease Contro; 5 SARS = Severe acute respiratory Syndrome; MERS = Middle east respiratory syndrome; PA/D = post-admisssion/discharge 
TABLE 5. QUALITY OF LIFE OUTCOMES

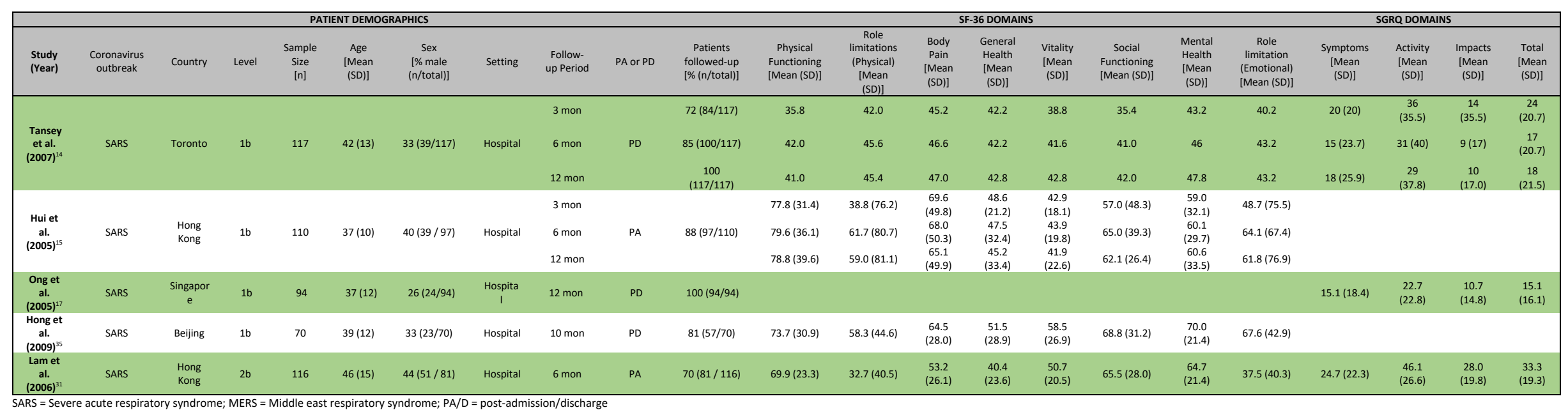

TABLE 6. MISCELLANEOUS OUTCOMES

\begin{tabular}{|c|c|c|c|c|c|c|c|c|c|c|c|c|}
\hline Study (Year) & $\begin{array}{c}\text { Coronavirus } \\
\text { Outbreak }\end{array}$ & System & Country & Level & $\begin{array}{l}\text { Sample } \\
\text { Size } \\
\text { [n] }\end{array}$ & $\begin{array}{c}\text { Age } \\
\text { [Mean } \\
\text { (SD)] }\end{array}$ & $\begin{array}{c}\text { Sex } \\
{[\% \text { male }} \\
[\text { (n/total) })]\end{array}$ & Setting & $\begin{array}{l}\text { Follow-up } \\
\text { Period }\end{array}$ & $\begin{array}{c}\text { Post- } \\
\text { admission } \\
\text { (PA) or } \\
\text { Post- } \\
\text { discharge } \\
\text { (PD) }\end{array}$ & $\begin{array}{l}\text { Patients } \\
\text { followed-up } \\
{[\%(n / t \text { totall) })]}\end{array}$ & Findings \\
\hline $\begin{array}{l}\text { Yuen et al. } \\
(2004)^{39}\end{array}$ & SARS & Ophthalmic & Hong Kong & $2 \mathrm{~b}$ & 45 & 39 (13) & $38(17 / 45)$ & Hospital & $\begin{array}{l}2 \text { mon } \\
3 \text { mon }\end{array}$ & PD & $\begin{array}{l}60(27 / 45) \\
33(15 / 45)\end{array}$ & $\begin{array}{l}\text { Elevated intraocular pressure: } 2 \text { patients (baseline), } 2 \text { patients ( } 2 \text { mon), , patient (3 mon) } \\
\text { No ocular manifestations of coronavirus were observed }\end{array}$ \\
\hline $\begin{array}{l}\text { Tsai et al. } \\
(2004)^{10}\end{array}$ & SARS & Neuromuscular & Taiwan & 4 & 4 & $46(7)$ & $25(1 / 4)$ & ICU & 3 mon & PA & $100(4 / 4)$ & $\begin{array}{l}\text { Series of patients developed distal-predominant weakness of } 4 \text { limbs, mild hyporeflexia and } \\
\text { hypesthesia in legs on day } 21,22,24 \text { and } 25 \text { with recoverry of muscle power in all patients at } 3 \text { months } \\
\text { follow-up }\end{array}$ \\
\hline
\end{tabular}


medRxiv preprint doi: https://doi.org/10.1101/2020.04.16.20067975; this version posted April 22, 2020. The copyright holder for this preprint (which was not certified by peer review) is the author/funder, who has granted medRxiv a license to display the preprint in perpetuity. All rights reserved. No reuse allowed without permission.

\section{Lung Function Outcomes}

18 studies (9 Level 1b, 4 Level 2b, 3 Level 3b, 2 Level 4 studies) reported lung function outcomes in CoV survivors of which 16 were included in the meta-analysis. Chen et al. (2006) ${ }^{13}$ only reported changes in symptoms without any report of lung function parameters which could be included in this meta-analysis. Zheng-Yu et al. $(2003)^{16}$ did not report standard deviations, hence, the data could not be used in the meta-analysis. Studies reporting prevalence of diffusing capacity of the lung for carbon monoxide (DLCO) (10 studies), forced expiratory volume in 1 second (FEV1) (6 studies), forced vital capacity (FVC) (5 studies) and total lung capacity (TLC) (4 studies) abnormalities were used to pool prevalence of each abnormality [Figure 2 and 3]. At 6 months, abnormalities in DLCO, FVC and TLC were more prevalent than abnormalities in FEV1. Most of these abnormalities improved after 6 months, however, the prevalence of DLCO impairment remained considerably high even 6 months post-infection, with pooled estimate of 24.35 (95\% confidence interval 11.05 to 45.46). Studies reporting mean value for DLCO (10 studies), FEV1(10 studies), FVC (10 studies), FEV1/FVC (6 studies), vital capacity (VC) (4 studies), diffusing capacity of the lung for carbon monoxide: Alveolar ventilation (DLCO:Va) (3 studies) and TLC (8 studies) were used to pool mean value for each abnormality up to and beyond 6 months [Figure 4]. The pooled estimates for none of these mean parameters were $<80 \%$ of predicted.

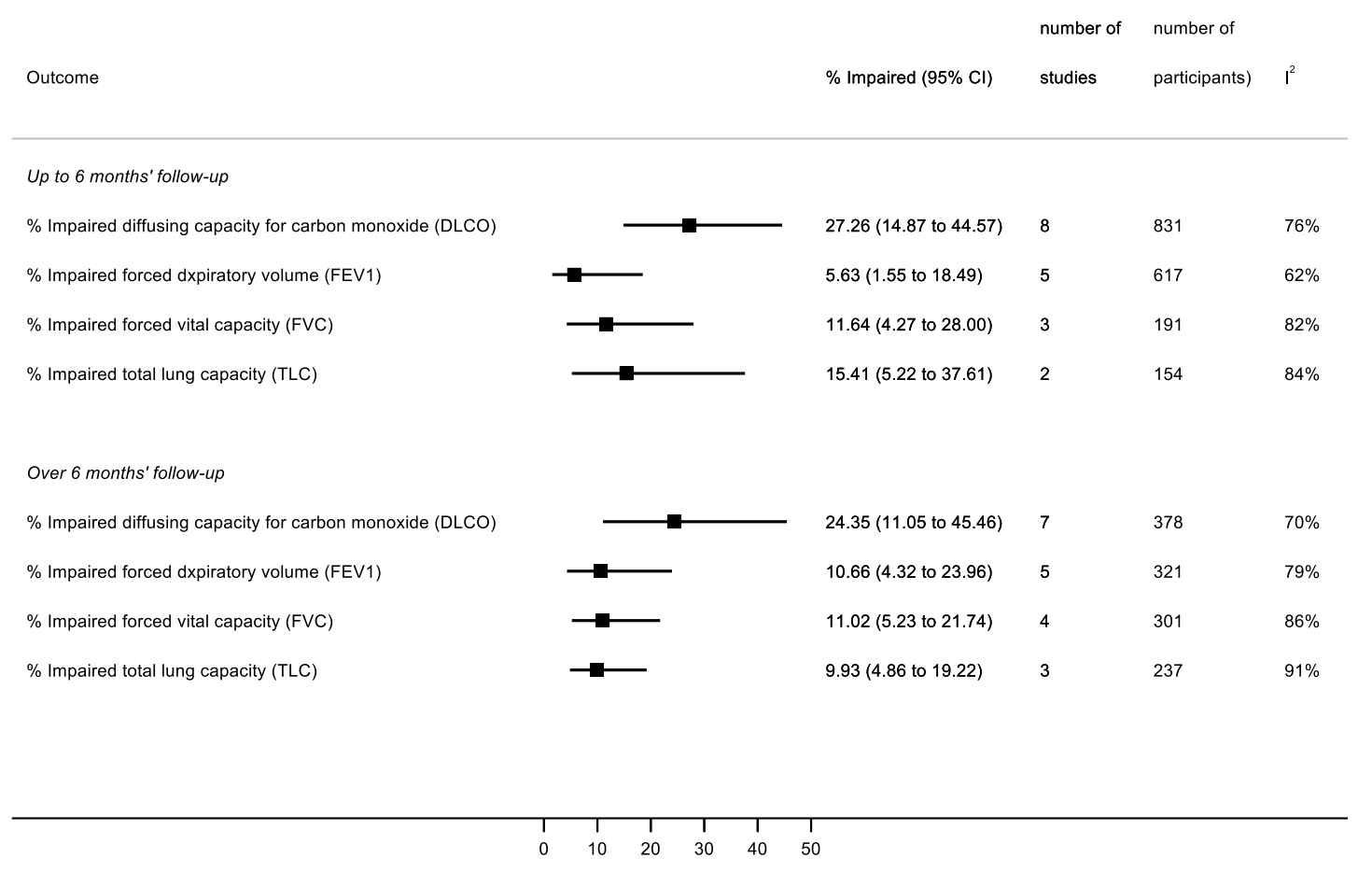

Figure 2. Summary plot showing pooled estimate of prevalence of different lung function abnormalities in CoV survivors up to 6 months (top) and over 6 months (bottom) 
medRxiv preprint doi: https://doi.org/10.1101/2020.04.16.20067975; this version posted April 22, 2020. The copyright holder for this preprint (which was not certified by peer review) is the author/funder, who has granted medRxiv a license to display the preprint in perpetuity. All rights reserved. No reuse allowed without permission.
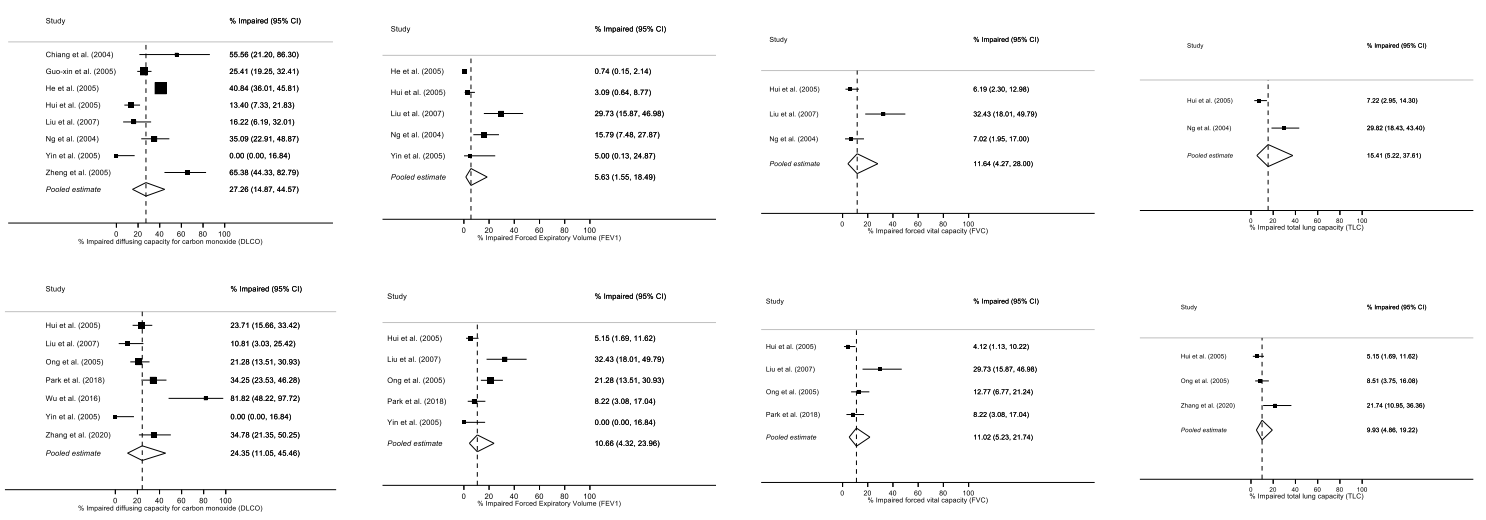

Figure 3. Forest plot showing pooled estimate of prevalence of different lung function abnormalities in CoV survivors up to 6 months (top) and over 6 months (bottom)
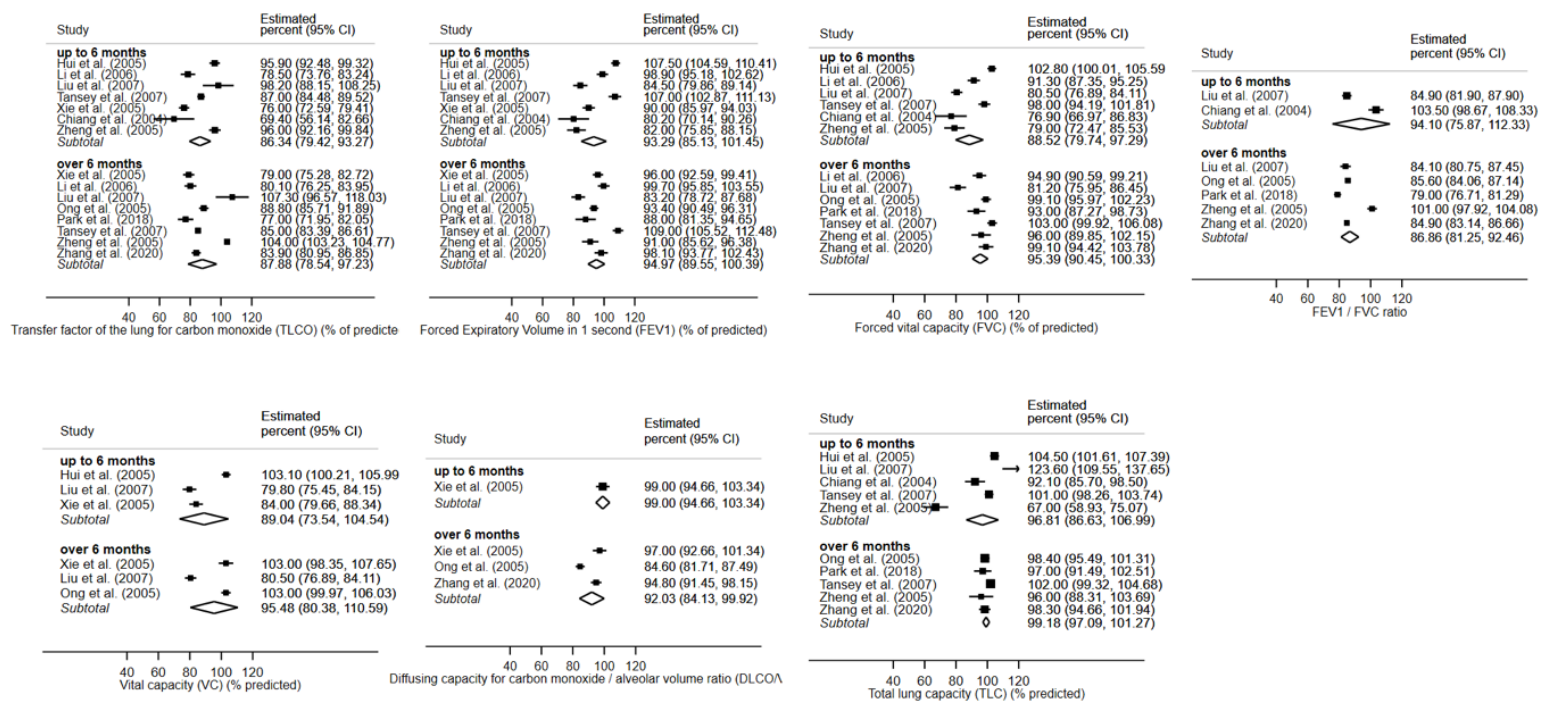

Figure 4. Forest plot showing pooled estimate of mean values of different lung function abnormalities in CoV survivors up to 6 months (top) and over 6 months (bottom) 
medRxiv preprint doi: https://doi.org/10.1101/2020.04.16.20067975; this version posted April 22, 2020. The copyright holder for this preprint (which was not certified by peer review) is the author/funder, who has granted medRxiv a license to display the preprint in perpetuity.

\section{Exercise Tolerance Outcomes}

5 studies (4 Level $1 \mathrm{~b}$ studies and 1 Level $2 \mathrm{~b}$ study) reported exercise tolerance outcomes in CoV survivors of which 4 were included in this meta-analysis [Figure 5]. Results from Ong et al. (2004) ${ }^{30}$ were not included because they only reported outcomes from cardiopulmonary exercise testing (CPET) and did not conduct 6-minute walking distance (6MWD). The pooled estimate of 6MWD for 3 studies reporting outcomes up to 6 months was 461.18 (95\% Confidence Interval 449.66 to 472.71 ). The 6MWD increased substantially after 6 months with pooled estimate of 533.00 (95\% Confidence Interval 449.66 to 472.71 ). Since $\sim 30 \mathrm{~m}$ is considered to be the minimal clinically important difference in $6 \mathrm{MWD}^{41}$, patients seem to improve significantly overtime. Unfortunately, data was not available regarding the 6MWD for participants before CoV infection and therefore there is no report of the number of patients with exercise tolerance lower than baseline.

$\begin{array}{ll}\text { Study } & \text { Mean distance }(95 \% \mathrm{Cl}) \\ \text { up to } 6 \text { months } & 468.00(442.00,494.00) \\ \text { Lam et al. (2006) } & 464.00(446.69,481.31) \\ \text { Hui et al. (2005) } & 454.00(434.79,473.21) \\ \text { Li et al. (2006) } & 461.18(449.66,472.71)\end{array}$

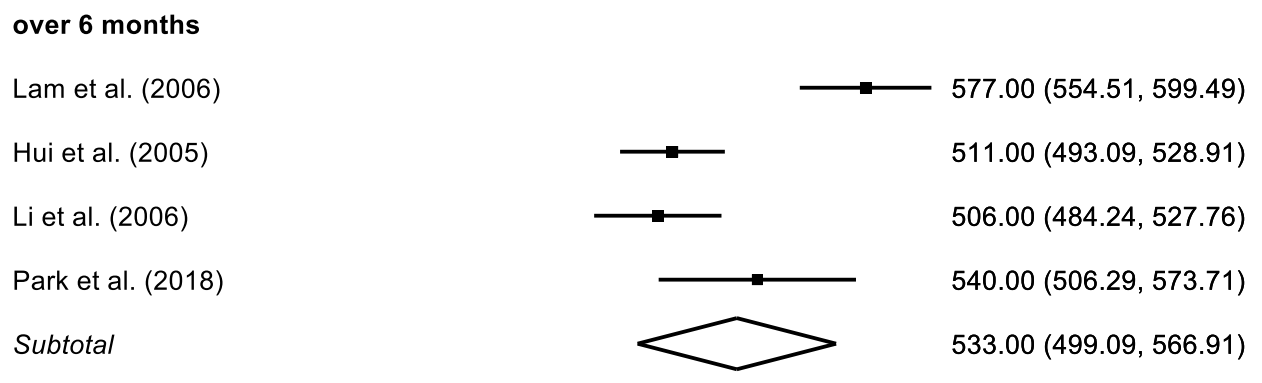

$\begin{array}{lcccc}1 & 1 & 1 & 1 \\ 400 & 450 & 500 & 550 & 600 \\ & & & \text { Six minute walk test distance }(\mathrm{m})\end{array}$

Figure 5. Summary plot showing pooled estimate of 6-minute walking distance in CoV survivors up to 6 months (top) and over 6 months (bottom) 
medRxiv preprint doi: https://doi.org/10.1101/2020.04.16.20067975; this version posted April 22, 2020. The copyright holder for this preprint (which was not certified by peer review) is the author/funder, who has granted medRxiv a license to display the preprint in perpetuity.

\section{Mental Health Outcomes}

6 studies ( 5 Level $1 \mathrm{~b}$ and 1 Level studies) reported psychological comorbidities in CoV survivors of which all 6 were included in the meta-analysis. All studies which reported prevalence of these psychological conditions had follow-up period of longer than 6 months. As a result, meta-analysis was conducted for prevalence beyond 6 months only [Figure 6 and 7]. The prevalence of different psychological conditions was substantially high with pooled estimates of $38.80 \%$ (95\% confidence interval 30.93 to 47.31 ) for post-traumatic stress disorder (PTSD), 33.20\% (95\% confidence interval 19.80 to 50.05 ) for depression and $30.04 \%$ (95\% confidence interval of 10.44 to 61.26 ) for anxiety [Figure 6 and Figure 7]. We could not perform meta-analysis on the mean scores for different psychological comorbidities because different scales were used by different studies to report these.
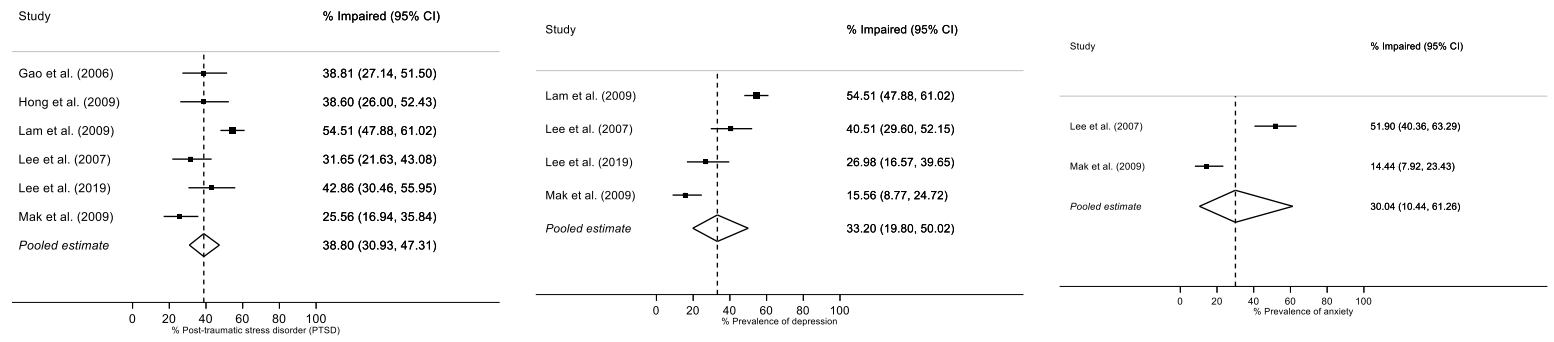

Figure 6. Forest plot showing pooled estimate of prevalence of different psychological conditions in CoV survivors over 6 months
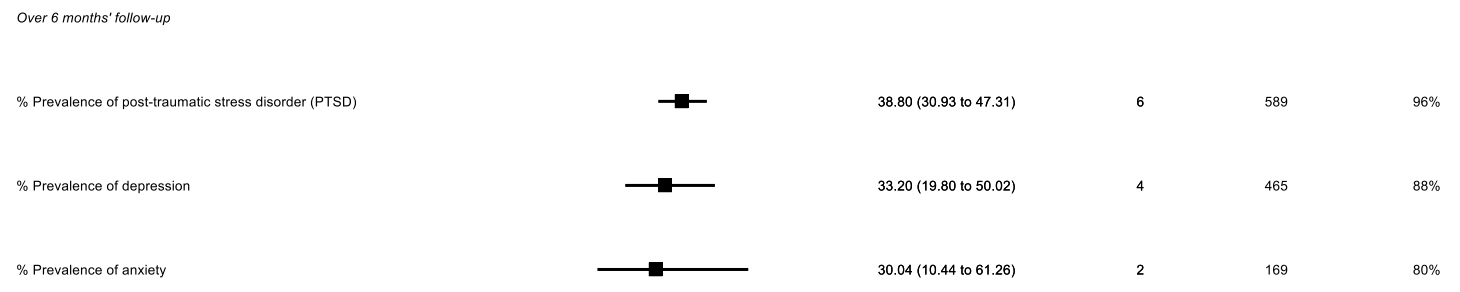

Figure 7. Summary plot showing pooled estimate of prevalence of different psychological conditions in CoV survivors over 6 months 
medRxiv preprint doi: https://doi.org/10.1101/2020.04.16.20067975; this version posted April 22, 2020. The copyright holder for this preprint (which was not certified by peer review) is the author/funder, who has granted medRxiv a license to display the preprint in perpetuity.

\section{Quality of Life Outcomes}

5 studies ( 4 Level $1 \mathrm{~b}$ studies and 1 Level $2 \mathrm{~b}$ study) reported quality of life outcomes in CoV survivors. Out of these, only 3 studies, which reported both mean and SD, were included in the meta-analysis of short form 36 health survey (SF-36) [Figure 8 and 9] and St George's Respiratory Questionnaire (SGRQ) [Figure 10] each. The pooled analysis showed that the mean score for all of the 8 domains of the SF36 were substantially lower in CoV survivors than normative values for people who are healthy as well as for people with chronic diseases derived from existing validated literature ${ }^{42}$ [Figure 9]. Domains which scored particularly lower than healthy individuals and those chronic conditions were role limitations due to physical and emotional health. There seems to be some improvement in these domains beyond 6 months, but the scores were still lower than healthy and chronic disease patients ${ }^{42}$.
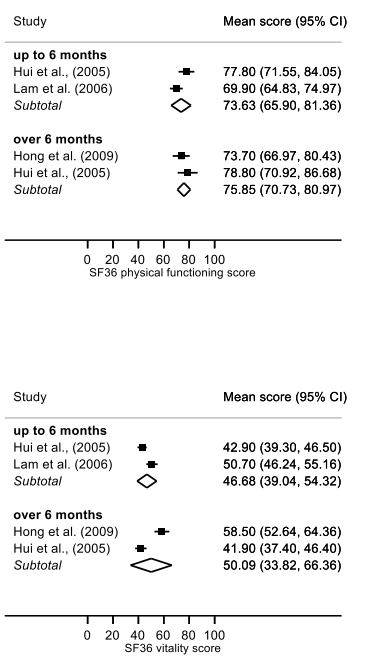
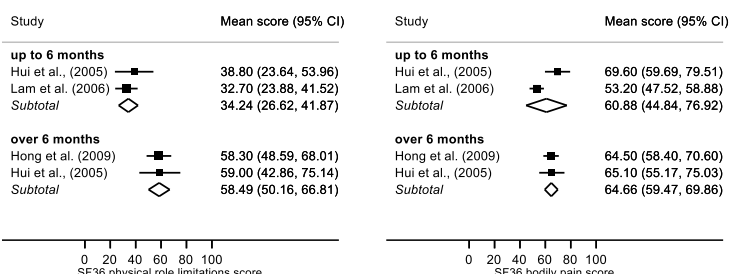

Study

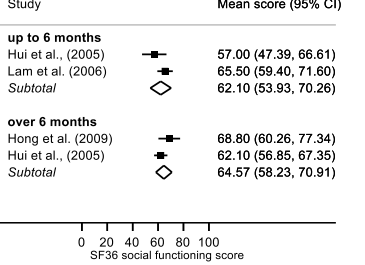

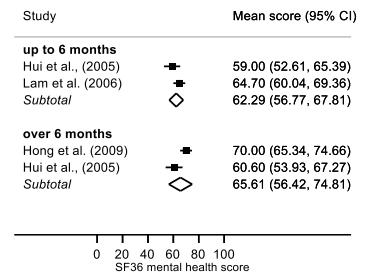

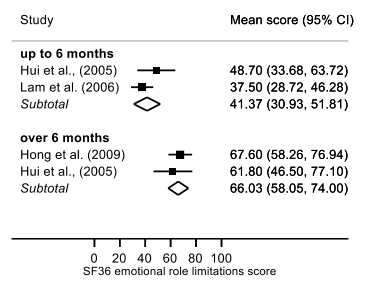

Figure 8. Forest plot showing pooled estimate of mean score for different domains of SF-36 in CoV survivors up to 6 months (top) and over 6 months (bottom) 
medRxiv preprint doi: https://doi.org/10.1101/2020.04.16.20067975; this version posted April 22, 2020. The copyright holder for this preprint (which was not certified by peer review) is the author/funder, who has granted medRxiv a license to display the preprint in perpetuity.

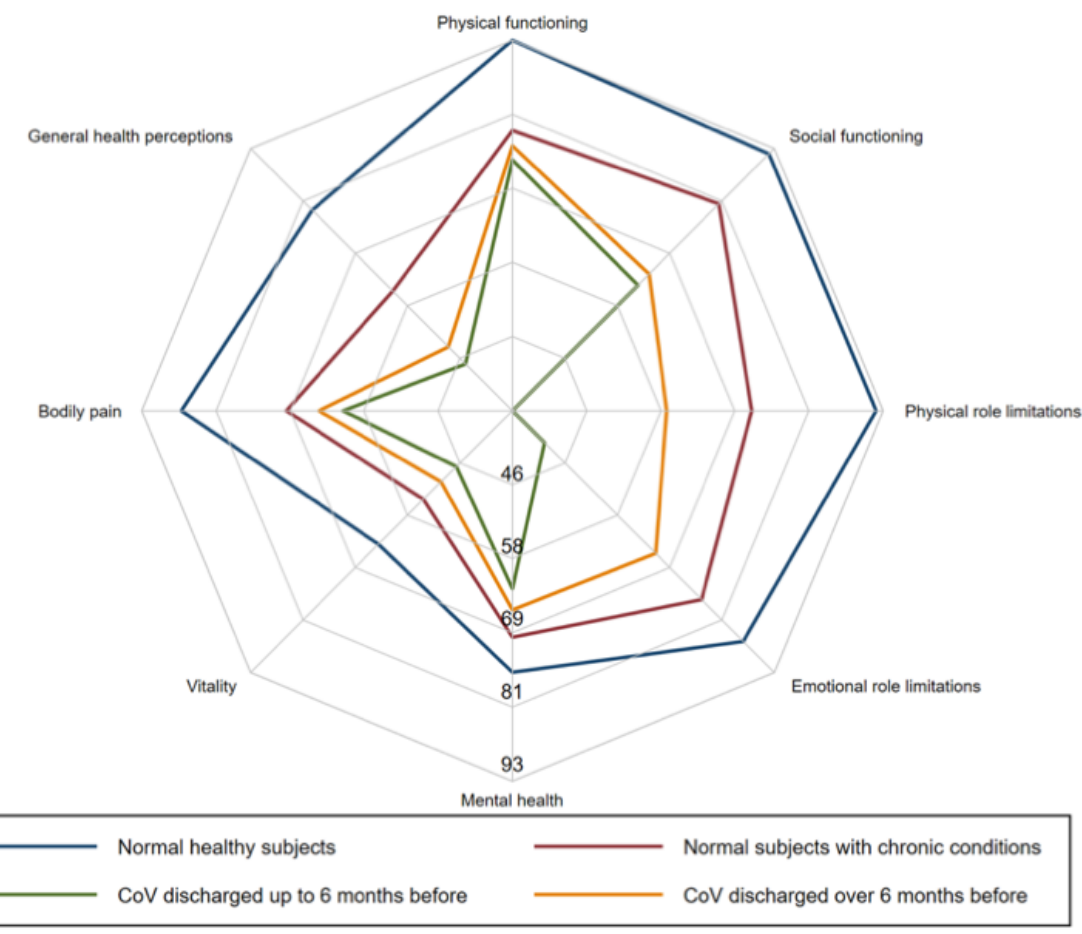

Figure 9. Radar plot showing pooled estimate of mean scores for different domains of SF-36 in CoV survivors up to 6 months (green) and over 6 months (orange) compared to healthy individuals (blue) and subjects with chronic conditions (red).
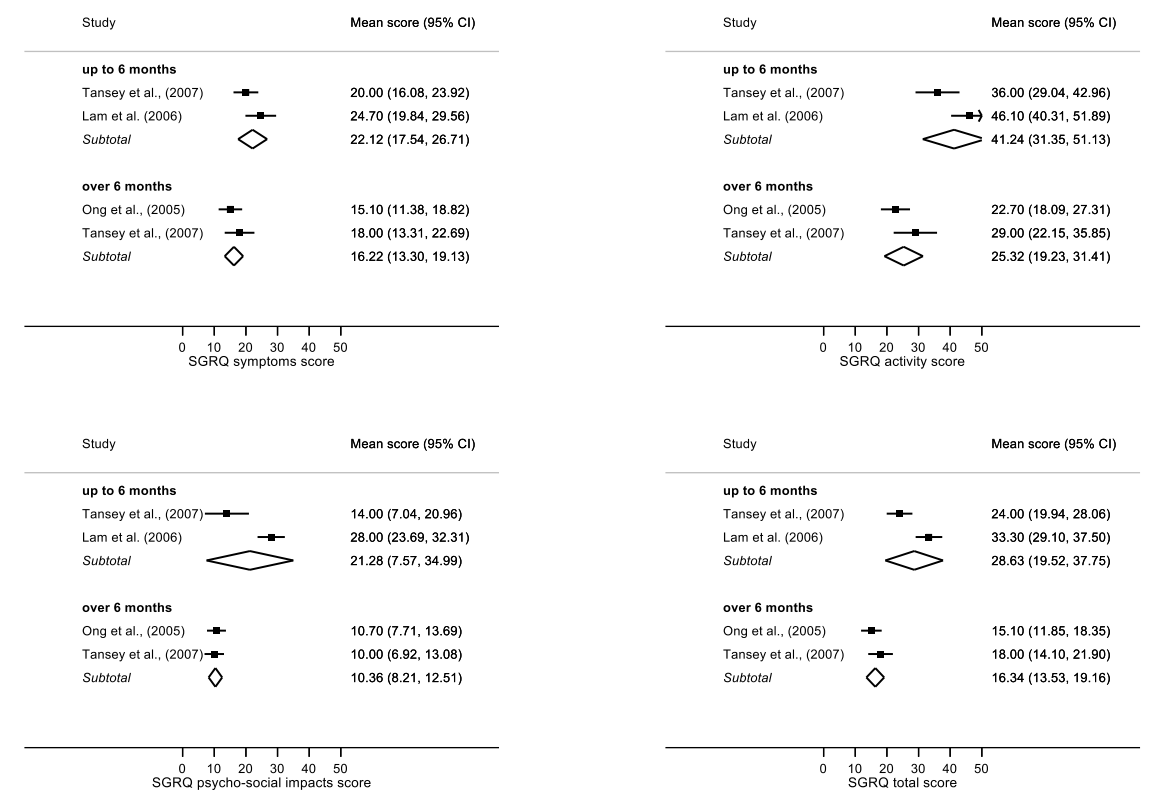

Figure 10. Forest plot showing pooled estimate of mean score for different domains of SGRQ in CoV survivors up to 6 months (top) and over 6 months (bottom) 
medRxiv preprint doi: https://doi.org/10.1101/2020.04.16.20067975; this version posted April 22, 2020. The copyright holder for this preprint (which was not certified by peer review) is the author/funder, who has granted medRxiv a license to display the preprint in perpetuity. All rights reserved. No reuse allowed without permission.

\section{Other Outcomes}

Other outcomes which have been followed-up in SARS patients have been reported in Table 6. The study by Yuen et al. (2004) $)^{39}$ found no eye pathologies in this cohort. The case-series by Tsai et al. $(2004)^{40}$ followed up patients who developed limb weakness related to critical illness neuropathy (CIN) and myopathy (CIM) and sensory deficits following infection. All these patients had a partial or full recovery of muscle power 3 months after admission.

\section{DISCUSSION}

The long-term complications of coronavirus infection are not well understood. The prevalence, severity and prognosis of these complications must be determined to plan the rehabilitation of survivors of the current COVID-19 pandemic. This systematic review collates these long-term complications seen following previous coronavirus outbreaks (SARS and MERS) in those who required hospitalisation or ICU stay. Our findings highlighted that the health-related quality of life (HRQoL), measured using SF-36, is considerably reduced in CoV survivors at 6 months postinfection, shows only slight improvement beyond 6 months and remains below normal population and those with chronic conditions [Figure 9]. As these SF-36 scores reflect impairment in physical, mental and social functioning of well-being, it is not surprising that the key areas of impairments identified in our systematic review were pulmonary dysfunction, reduced exercise tolerance and psychological problems.

Respiratory compromise is one of the key physical issues in survivors. The impairment is mainly restrictive in nature with predominance of abnormalities in DLCO, VC and TLC compared to FEV1, thereby, supporting the etiopathology of acute respiratory distress syndrome with parenchymal infiltration caused by the infection. Even though lung function improves over time, the results from our meta-analysis showed that reduction in DLCO may still be present in 11 to $45 \%$ of CoV survivors at 12 months. This is consistent with CT findings of other studies which have reported that pulmonary fibrosis can persist up to 7 years ${ }^{29}$. Pulmonary rehabilitation has been shown to improve QoL in other patients with fibrosis ${ }^{43}$ but it is unknown whether this would be effective in COVID-19 survivors.

CoV survivors had reduced aerobic capacity with peak oxygen uptake (VO2max) testing showing impairments in $41 \%$ of patients at 3 months ${ }^{30}$. This could be due to circulatory limitation, muscle weakness, critical illness neuropathy and myopathy (CINM) and deconditioning ${ }^{30}$. The 6MWD is also reduced at 3 months and slowly improves by 12 months ${ }^{44,45}$. We know from other literature that such chronic weakness may be present in patients even 5 years after ICU admission, therefore, rehabilitation needs of these patients can be prolonged ${ }^{46}$. Early rehabilitation combining mobilisation 
medRxiv preprint doi: https://doi.org/10.1101/2020.04.16.20067975; this version posted April 22, 2020. The copyright holder for this preprint (which was not certified by peer review) is the author/funder, who has granted medRxiv a license to display the preprint in perpetuity. All rights reserved. No reuse allowed without permission.

with strengthening exercises may improve exercise tolerance in these patient groups as it has substantial evidence for improving weakness and functional independence in $\mathrm{CINM}^{47}$.

Our meta-analysis showed that around a third of CoV survivors may have psychological conditions such as PTSD, depression and anxiety beyond 6 months. These estimates are much higher than the prevalence of these conditions reported as part of post-ICU syndrome in medical and surgical patients ${ }^{48}$. This indicates that the long-lasting mental health impact is not from serious illness alone, but also from factors such as fear ${ }^{49}$, stigma ${ }^{37}$ and quarantine ${ }^{50}$, all of which also apply to COVID-19 ${ }^{51}$. The neuropsychiatric aspects of CoV infections are not very well known yet and priorities and strategies for mental health science research have already been set out ${ }^{52}$.

SF-36 scores for role limitations in CoV survivors were particularly low compared to healthy individuals. Tansey et al. $(2007)^{14}$ reported that $17 \%$ CoV survivors had not returned to their previous level of working even at 1 year post-infection. Many of the symptoms experienced by CoV survivors could be responsible for such reduced social functioning. Fatigue was reported to be present in at least a third of the patients in two studies with a follow-up period of 18 months $s^{36}$ and 40 months $s^{37}$ each. Pain disorders were followed-up in one study which reported it to be present in about one-third of patients ${ }^{37}$.

The main strength of this study is that it highlights multiple long-term biopsychosocial impairments which may hinder return to pre-infection functional status. This is the first systematic review and meta-analysis on this topic as far as we are aware. Unlike a previous review from $2003^{7}$, we investigated long-term outcomes from major SARS and MERS outbreaks this century. We have tried to capture the various aspects of well-being and health-related quality of life in CoV survivors. There are understandably no studies on the long-term effects of COVID-19 as the outbreak was first reported only in Dec 2019. Considering SARS-CoV-2 belongs to the same virus family and has led to a more rapid spread with greater mortality worldwide ${ }^{1}$, the aftereffects are predicted to be similar, if not more profound and demanding of healthcare resources long term. For example, the widely reported prevalence of coagulopathy and thrombotic disease in COVID-19 patients may result in new end-organ complications and respiratory recovery could conceivably be affected ${ }^{53}$, thereby, leading to worse outcomes long-term.

Finally, there was also a paucity of information following up SARS and MERS survivors. Many studies had a small sample size and some outcomes could not be quantified because of limited number of studies reporting these. There was substantial heterogeneity, with almost all I-squared estimates $>50 \%$. We were unable to formally explore sources of this heterogeneity because of the small 
medRxiv preprint doi: https://doi.org/10.1101/2020.04.16.20067975; this version posted April 22, 2020. The copyright holder for this preprint (which was not certified by peer review) is the author/funder, who has granted medRxiv a license to display the preprint in perpetuity. All rights reserved. No reuse allowed without permission.

numbers, but these could include study-level differences in mean ages, gender, differences between SARS and MERS outbreaks, referral pathways between regions and study design.

Differences in outcomes between ICU and non-ICU patients remain unclear. Whilst one study identified that lung function parameters like FVC and DLCO were comparatively lower in ICU group ${ }^{15}$, another reported no significant difference between the two groups ${ }^{30}$. Further reporting of outcomes in ICU CoV survivors would be crucial as muscle weakness developed during ICU admissions has been associated with substantial impairments in physical function and quality of life ${ }^{54}$. Therefore, coronavirus survivors who required ICU will likely have even worse outcomes.

The global community of rehabilitation and mental healthcare services need to address the long-term complications identified in this review very early in the COVID-19 pandemic recovery phase. Acute rehabilitation during hospital stay requires active involvement of multidisciplinary teams to ensure the physical, psychological and social aspects are met. Post-acute early rehabilitation in the first 3 months after discharge is critical to prevent emerging issues such as reduced exercise tolerance and depression. Long-term rehabilitation must be an ongoing process to ensure individual function and biopsychosocial profiles are restored as much as possible so these individuals can return to previous societal roles and start contributing successfully to economies. This will determine whether the healthcare services around the globe have successfully managed the long-term impact of this pandemic.

\section{REFERENCES}

1. Peeri NC, Shrestha N, Rahman MS, Zaki R, Tan Z, Bibi S, et al. The SARS, MERS and novel coronavirus (COVID-19) epidemics, the newest and biggest global health threats: what lessons have we learned? Int J Epidemiol [Internet]. 2020 Feb 22; Available from: https://doi.org/10.1093/ije/dyaa033

2. Gorbalenya AE, Baker SC, Baric RS, de Groot RJ, Drosten C, Gulyaeva AA, et al. The species Severe acute respiratory syndrome-related coronavirus: classifying 2019-nCoV and naming it SARS-CoV-2. Nat Microbiol [Internet]. 2020;5(4):536-44. Available from: https://doi.org/10.1038/s41564-020-0695-z

3. Zhu N, Zhang D, Wang W, Li X, Yang B, Song J, et al. A Novel Coronavirus from Patients with Pneumonia in China, 2019. N Engl J Med [Internet]. 2020 Jan 24;382(8):727-33. Available from: https://doi.org/10.1056/NEJMoa2001017

4. WHO. WHO Director-General's opening remarks at the media briefing on COVID-19-11 March 2020 [Internet]. 2020 [cited 2020 Apr 12]. Available from: 
medRxiv preprint doi: https://doi.org/10.1101/2020.04.16.20067975; this version posted April 22, 2020. The copyright holder for this preprint (which was not certified by peer review) is the author/funder, who has granted medRxiv a license to display the preprint in perpetuity. All rights reserved. No reuse allowed without permission.

https://www.who.int/dg/speeches/detail/who-director-general-s-opening-remarks-at-themedia-briefing-on-covid-19---11-march-2020

5. World Health Organization. Coronavirus Disease (COVID-19) Pandemic [Internet]. 2020 [cited 2020 Apr 15]. Available from: https://www.who.int/emergencies/diseases/novelcoronavirus-2019

6. Verity R, Okell LC, Dorigatti I, Winskill P, Whittaker C, Imai N, et al. Estimates of the severity of coronavirus disease 2019: a model-based analysis. Lancet Infect Dis [Internet]. 2020 Apr 11; Available from: https://doi.org/10.1016/S1473-3099(20)30243-7

7. Chan KS, Zheng JP, Mok YW, Li YM, Liu YN, Chu CM, et al. SARS: Prognosis, outcome and sequelae. Respirology [Internet]. 2003;8 Suppl:S36-40. Available from: http://ovidsp.ovid.com/ovidweb.cgi?T=JS\&PAGE=reference\&D=med5\&NEWS=N\&AN=150181 32

8. OCEBM. Oxford Centre of Evidence Based Medicine Level of Evidence Tool 2009 [Internet]. 2009 [cited 2020 Apr 11]. Available from: https://www.cebm.net/2009/06/oxford-centreevidence-based-medicine-levels-evidence-march-2009/

9. DerSimonian R, Laird N. Meta-analysis in clinical trials. Control Clin Trials. 1986;7(3):177-88.

10. Higgins JPT, Thompson SG, Deeks JJ, Altman DG. Measuring inconsistency in meta-analyses. Br Med J. 2003;327(7414):557-60.

11. StataCorp. 2017. Stata: Release 15. Statistical Software. College Station, TX: StataCorp LLC.

12. He ZY. A follow-up study of the lung function and the chest CT changes in medical staff with severe acute respiratory syndrome in Beijing. Chinese J Tuberc Respir Dis. 2005;28(1):10-2.

13. Chen JH, Ma DQ, He W, Jin EH, Zhang J, Zhong ZH. Follow-up study of chest CT manifestations of patients with severe acute respiratory syndrome. Chinese J Radiol [Internet]. 2006;40(11):1161-5. Available from: http://ovidsp.ovid.com/ovidweb.cgi?T=JS\&PAGE=reference\&D=emed9\&NEWS=N\&AN=44921 406

14. Tansey CM, Louie M, Loeb M, Gold WL, Muller MP, De Jager JA, et al. One-year outcomes and health care utilization in survivors of severe acute respiratory syndrome. Arch Intern Med. 2007;167(12):1312-20.

15. Hui DS, Wong KT, Ko FW, Tam LS, Chan DP, Woo J, et al. The 1-year impact of severe acute 
medRxiv preprint doi: https://doi.org/10.1101/2020.04.16.20067975; this version posted April 22, 2020. The copyright holder for this preprint (which was not certified by peer review) is the author/funder, who has granted medRxiv a license to display the preprint in perpetuity. All rights reserved. No reuse allowed without permission.

respiratory syndrome on pulmonary function, exercise capacity, and quality of life in a cohort of survivors. Chest [Internet]. 2005;128(4):2247-61. Available from: http://ovidsp.ovid.com/ovidweb.cgi?T=JS\&PAGE=reference\&D=med6\&NEWS=N\&AN=162368 81

16. Zheng-yu J, Hui Y, Wei-hong Z, Yun W, Ji-Xiang L, Wen-bin M, et al. Thoracic high resolution CT findings of 100 SARS patients in convalescent period. Acta Acad Med Sin. 2003;25(5):5125.

17. Ong K-C, Ng AW-K, Lee LS-U, Kaw G, Kwek S-K, Leow MK-S, et al. 1-year pulmonary function and health status in survivors of severe acute respiratory syndrome. Chest [Internet]. 2005;128(3):1393-400. Available from: http://ovidsp.ovid.com/ovidweb.cgi?T=JS\&PAGE=reference\&D=med6\&NEWS=N\&AN=161627 34

18. Park WB, Jun KI, Kim G, Choi J-P, Rhee J-Y, Cheon S, et al. Correlation between Pneumonia Severity and Pulmonary Complications in Middle East Respiratory Syndrome. J Korean Med Sci [Internet]. 2018;33(24):e169. Available from:

http://ovidsp.ovid.com/ovidweb.cgi?T=JS\&PAGE=reference\&D=emed19\&NEWS=N\&AN=6242 21590

19. Li TS, Gomersall CD, Joynt GM, Chan DPSS, Leung P, Hui DSCC. Long-term outcome of acute respiratory distress syndrome caused by severe acute respiratory syndrome (SARS): an observational study. Crit care Resusc [Internet]. 2006;8(4):302-8. Available from: http://ovidsp.ovid.com/ovidweb.cgi?T=JS\&PAGE=reference\&D=med6\&NEWS=N\&AN=172272 66

20. Liu Y-X, Ye Y-P, Zhang P, Chen J, Ye H, He Y-H, et al. Changes in pulmonary function in SARS patients during the three-year convalescent period. Zhongguo Wei Zhong Bing Ji Jiu Yi Xue [Internet]. 2007;19(9):536-8. Available from:

http://ovidsp.ovid.com/ovidweb.cgi?T=JS\&PAGE=reference\&D=med6\&NEWS=N\&AN=177678 22

21. Ng CK, Chan JWM, Kwan TL, To TS, Chan YH, Ng FYY, et al. Six month radiological and physiological outcomes in severe acute respiratory syndrome (SARS) survivors. Thorax. 2004;59(10):889-91.

22. Zheng Z-G, Chen R, Wu H, Liu X, He W, Xu Y, et al. Changes in pulmonary function in severe acute respiratory syndrome patients during convalescent period. Chinese Crit Care Med. 
medRxiv preprint doi: https://doi.org/10.1101/2020.04.16.20067975; this version posted April 22, 2020. The copyright holder for this preprint (which was not certified by peer review) is the author/funder, who has granted medRxiv a license to display the preprint in perpetuity.

2015;17(6):329-31.

23. Zhang P, Li J, Liu H, Han N, Ju J, Kou Y, et al. Long-term consequences in lung and bone associated with hospital-acquired severe acute respiratory syndrome: a 15-year follow-up from a prospective cohort study. Lancet [Internet]. 2020;8(Supplement 1):S11. Available from:

http://ovidsp.ovid.com/ovidweb.cgi?T=JS\&PAGE=reference\&D=emed19\&NEWS=N\&AN=2001 217701

24. Chiang CH, Shih JF, Su WJ, Perng RP. Eight-month prospective study of 14 patients with hospital-acquired severe acute respiratory syndrome. Mayo Clin Proc. 2004;79(11):1372-9.

25. Xie L, Liu Y, Fan B, Xiao Y, Tian Q, Chen L, et al. Dynamic changes of serum SARS-Coronavirus IgG, pulmonary function and radiography in patients recovering from SARS after hospital discharge. Respir Res [Internet]. 2005;6:5. Available from: http://respiratoryresearch.com/content/6/1/5

26. Guoxin M, Lixin X, Baoxing F, Xinli D, Xiaoling Q, Youning L. Association of T-lymphocyte subpopulation level with age, gender, rehabilitative duration and lung diffusion function in patients with severe acute respiratory syndrome during rehabilitative period: A follow-up of 227 cases. Chinese J Clin Rehabil [Internet]. 2005;9(7):90-2. Available from: http://ovidsp.ovid.com/ovidweb.cgi?T=JS\&PAGE=reference\&D=emed9\&NEWS=N\&AN=40846 744

27. Wong K, Antonio GE, Hui DSC, Ho C, Chan P, Ng W, et al. Severe Acute Respiratory Syndrome Thin-Section Computed Tomography Features, Temporal Changes, and Clinicoradiologic Correlation During the Convalescent Period. Lung. 2004;28(6):790-5.

28. Yin C, Wang C, Wen Y, Jiang L, Lu Q, Li J, et al. Prospective 2-year clinical study of patients with positive IgG-antibodies after recovering from severe acute respiratory syndrome. Chinese Crit Care Med. 2005;17(12):740-2.

29. Wu X, Dong D, Ma D, X. W, D. D. Thin-section computed tomography manifestations during convalescence and long- term follow-up of patients with severe acute respiratory syndrome (SARS). Med Sci Monit [Internet]. 2016;22:2793-9. Available from: http://www.medscimonit.com/download/index/idArt/896985

30. Ong K-CC, Ng AWKW-K, Lee LS-USU, Kaw G, Kwek S-KK, Leow MKSK-S, et al. Pulmonary function and exercise capacity in survivors of severe acute respiratory syndrome. Eur Respir J 
medRxiv preprint doi: https://doi.org/10.1101/2020.04.16.20067975; this version posted April 22, 2020. The copyright holder for this preprint (which was not certified by peer review) is the author/funder, who has granted medRxiv a license to display the preprint in perpetuity. All rights reserved. No reuse allowed without permission.

[Internet]. 2004;24(3):436-42. Available from:

http://ovidsp.ovid.com/ovidweb.cgi?T=JS\&PAGE=reference\&D=med5\&NEWS=N\&AN=153587

03

31. Lam SP, Tsui E, Chan KS, Lam CL, So HP, S.P. L, et al. The validity and reliability of the functional impairment checklist (FIC) in the evaluation of functional consequences of severe acute respiratory distress syndrome (SARS). Qual Life Res [Internet]. 2006;15(2):217-31.

Available from:

http://ovidsp.ovid.com/ovidweb.cgi?T=JS\&PAGE=reference \&D=emed9\&NEWS=N\&AN=43230 113

32. Lee AM, Wong JGWS, McAlonan GM, Cheung V, Cheung C, Sham PC, et al. Stress and psychological distress among SARS survivors 1 year after the outbreak. Can J Psychiatry [Internet]. 2007;52(4):233-40. Available from:

http://ovidsp.ovid.com/ovidweb.cgi?T=JS\&PAGE=reference\&D=med6\&NEWS=N\&AN=175003 04

33. Mak IWC, Chu CM, Pan PC, Yiu MGC, Chan VL. Long-term psychiatric morbidities among SARS survivors. Gen Hosp Psychiatry [Internet]. 2009;31(4):318-26. Available from:

http://dx.doi.org/10.1016/j.genhosppsych.2009.03.001

34. Gao H, Hui W, Lan X. A follow-up study of post-traumatic stress disorder of SARS patients after discharge. Chinese J Rehabil Med. 2006;21(11):3-5.

35. Hong X, Currier GW, Zhao X, Jiang Y, Zhou W, Wei J. Posttraumatic stress disorder in convalescent severe acute respiratory syndrome patients: a 4-year follow-up study. Gen Hosp Psychiatry [Internet]. 2009;31(6):546-54. Available from:

http://ovidsp.ovid.com/ovidweb.cgi?T=JS\&PAGE=reference\&D=med7\&NEWS=N\&AN=198922 13

36. Lee SH, Shin HS, Park HY, Kim JL, Lee JJ, Lee H, et al. Depression as a mediator of chronic fatigue and post-traumatic stress symptoms in middle east respiratory syndrome survivors. Psychiatry Investig. 2019;16(1):59-64.

37. Lam $M$, Wing $Y, Y u M$, Leung $C$, Ma R, Kong $A$, et al. Mental morbidities and chronic fatigue in severe acute respiratory syndrome survivors: Long-term follow-up. Arch Intern Med [Internet]. 2009 Dec 14;169(22):2142-7. Available from:

http://search.ebscohost.com/login.aspx?direct=true\&AuthType=ip,shib\&db=jlh\&AN=105271 209\&site=ehost-live 
medRxiv preprint doi: https://doi.org/10.1101/2020.04.16.20067975; this version posted April 22, 2020. The copyright holder for this preprint

(which was not certified by peer review) is the author/funder, who has granted medRxiv a license to display the preprint in perpetuity. All rights reserved. No reuse allowed without permission.

38. Tansey CM, Louie M, Loeb M, Gold WL, Muller MP, de Jager J, et al. One-year outcomes and health care utilization in survivors of severe acute respiratory syndrome. Arch Intern Med [Internet]. 2007;167(12):1312-20. Available from:

http://ovidsp.ovid.com/ovidweb.cgi?T=JS\&PAGE=reference\&D=med6\&NEWS=N\&AN=175921 06

39. Yuen KSC, Chan WM, Fan DSP, Chong KKL, Sung JJY, Lam DSC. Ocular screening in severe acute respiratory syndrome. Am J Ophthalmol. 2004;137(4):773-4.

40. Tsai LK, Hsieh ST, Chao CC, Chen YC, Lin YH, Chang SC, et al. Neuromuscular disorders in severe acute respiratory syndrome. Arch Neurol. 2004;61(11):1669-73.

41. Holland AE, Nici L. The return of the minimum clinically important difference for 6-minutewalk distance in chronic obstructive pulmonary disease. American Journal of Respiratory and Critical Care Medicine. 2013.

42. Bentley C. Adults of working age (ages 25-64). Heal Inequal Appl Public Heal Res to Policy Pract. 2014;306(Sf 36):97-9.

43. Hanada M, Kasawara K, Mathur S, Rozenberg D, Kozu R, Reid D. Aerobic and Breathing Exercises Improve Dyspnea, Exercise Capacity and Quality of Life in Idiopathic Pulmonary Fibrosis Patients: Systematic Review and Meta-Analysis. J Thorac Dis. 2020;12(3):1041-55.

44. Hui DS, Wong KT, Ko FW, Tam LS, Chan DP, Woo J, et al. The 1-year impact of severe acute respiratory syndrome on pulmonary function, exercise capacity, and quality of life in a cohort of survivors. Chest [Internet]. 2005;128(4):2247-61. Available from:

http://ovidsp.ovid.com/ovidweb.cgi?T=JS\&PAGE=reference\&D=med6\&NEWS=N\&AN=162368 81

45. Li TS, Gomersall CD, Joynt GM, Chan DPS, Leung P, Hui DSC. Long-term outcome of acute respiratory distress syndrome caused by severe acute respiratory syndrome (SARS): an observational study. Crit Care Resusc [Internet]. 2006;8(4):302-8. Available from: http://ovidsp.ovid.com/ovidweb.cgi?T=JS\&PAGE=reference\&D=med6\&NEWS=N\&AN=172272 66

46. Ricks E. Critical illness polyneuropathy and myopathy: a review of evidence and the implications for weaning from mechanical ventilation and rehabilitation. Physiotherapy. 2007;93(2):151-6.

47. Zink W, Kollmar R, Schwab S. Critical illness polyneuropathy and myopathy in the intensive 
medRxiv preprint doi: https://doi.org/10.1101/2020.04.16.20067975; this version posted April 22, 2020. The copyright holder for this preprint

(which was not certified by peer review) is the author/funder, who has granted medRxiv a license to display the preprint in perpetuity.

All rights reserved. No reuse allowed without permission.

care unit. Nat Rev Neurol [Internet]. 2009;5(7):372-9. Available from:

https://doi.org/10.1038/nrneurol.2009.75

48. Herridge MS, Chu LM, Matte A, Tomlinson G, Chan L, Thomas C, et al. The RECOVER program: Disability risk groups and 1-year outcome after 7 or more days of mechanical ventilation. Am J Respir Crit Care Med. 2016;

49. Bener A, Al-Khal A. Knowledge, attitude and practice towards SARS. J R Soc Promot Health. 2004;

50. Reynolds DL, Garay JR, Deamond SL, Moran MK, Gold W, Styra R. Understanding, compliance and psychological impact of the SARS quarantine experience. Epidemiol Infect. 2008 Jul;136(7):997-1007.

51. WHO. Mental health and psychosocial considerations during the COVID-19 outbreak [Internet]. 2020 [cited 2020 Apr 15]. Available from: https://www.who.int/docs/defaultsource/coronaviruse/mental-health-considerations.pdf

52. Holmes EA, Connor RCO, Perry VH, Tracey I, Wessely S, Arseneault L, et al. Multidisciplinary research priorities for the COVID-19 pandemic : a call for action for mental health science. The Lancet Psychiatry [Internet]. 2020;0366(20):1-14. Available from: http://dx.doi.org/10.1016/S2215-0366(20)30168-1

53. Tang N, Bai H, Chen X, Gong J, Li D, Sun Z. Anticoagulant treatment is associated with decreased mortality in severe coronavirus disease 2019 patients with coagulopathy. J Thromb Haemost. 2020;

54. Fan E, Dowdy DW, Colantuoni E, Mendez-Tellez PA, Sevransky JE, Shanholtz C, et al. Physical complications in acute lung injury survivors: A two-year longitudinal prospective study. Crit Care Med. 2014;42(4):849-59. 